\title{
Modelación basada en agentes en el sistema pensional colombiano. \\ Una aproximación desde el mercado laboral y la dinámica poblacional ${ }^{\star}$
}

\section{Agent-based modeling in Colombia's pension system.}

An approach from the labour market

and population dynamics

Nancy del Carmen Quiceno Cárdenas**

Recibido: 14 de mayo de 2014

Revisado: 20 de junio de 2014

Aprobado: 11 de septiembre de 2014

\section{Resumen}

Este artículo corresponde a un estudio del sistema pensional colombiano (SPC), reglamentado por la Ley 100 de 1993. Por medio de un Modelo Basado en Agentes (MBA), se analizan características propias de los agentes en el SPG con respecto a su

* Cómo citar este artículo: Quiceno, N. (2014). Modelación basada en agentes en el sistema pensional colombiano. Una aproximación desde el mercado laboral y la dinámica poblacional. Revista CIFE, 16(24), 119-162.

** Magister en ciencias económicas, Universidad Santo Tomás. Especialista en gestión de riesgos financieros, Universidad Sergio Arboleda. Matemática de la Universidad Nacional de Colombia. Correo electrónico: nancy. quiceno@gmail.com 
articulación al mercado laboral y a la dinámica poblacional, quienes asumen uno de los roles en el sistema pensional en procura de lograr su pensión. Las probabilidades de los agentes de adoptar un régimen están condicionadas por las características del sistema pensional y por variables económicas, sociales y del sistema político; las variables pueden cambiar junto a la evolución del sistema pensional, debido a las acciones colectivas de los agentes y a las decisiones de política pública.

Palabras clave: protección social, simulación basada en agentes, economía evolucionista, régimen pensional

Glasificación JEL: B52, C69, G73, J2

\section{Abstract}

This document is a study of the Colombian Pension System (CPS) regulated by Law 100 of 1993. Using an agent-based model (ABM), it analyzes the characteristics of agents in the CPS regarding their articulation to the labour market and population dynamics; they whom assume one of the roles in the pension system in order to achieve their pension. The odds for agents to adopt a regime are conditioned by the characteristics of the pension system and economical, social and political system variables which can change along with the evolution of the pension system, due to collective actions of agents and public policy decisions.

Keywords: Social protection, agent-based modeling, evolutionary economics, pension regime

Classification JEL: B52, C69, C73, J2 


\section{Introducción}

Los sistemas pensionales obligatorios formalizan el acuerdo social por el cual los trabajadores reciben una suma de dinero durante su vejez, de acuerdo con un ahorro ejecutado durante su tiempo productivo. Esquemáticamente, el sistema funciona de la siguiente forma: un porcentaje de los salarios de cada trabajador, determinado por la normatividad, se deposita como un ahorro obligatorio; el acumulado de todos los trabajadores se invierte en el mercado de valores para generar una rentabilidad regulada por el Estado. En el momento de jubilación, la suma de los aportes del trabajador y los rendimientos financieros se entregan al trabajador en una serie de pagos mensuales, siempre y cuando él cumpla con las condiciones legales de edad y tiempo de aporte, entre otras.

La evolución de las características de la mano de obra, la composición social, la distribución salarial y la dinámica poblacional, entre otras, han conducido a reformas en los sistemas pensionales para favorecer el cumplimiento de sus objetivos. Colombia reformó el sistema general de pensiones en 1993; reforma que se encuentra reglamentada en la Ley 100 del mismo año, y en los últimos veinte años se le han hecho varias modificaciones con el fin de aumentar la cobertura y la financiación del sistema.

La forma en que el sistema pensional opera en su interior e interactúa en la economía y en la sociedad se ha transformado, junto con el mercado laboral y la dinámica de la población. La presencia de la informalidad en la economía, más precisamente en el mercado laboral, sugiere la necesidad de adaptar los esquemas teóricos y metodológicos para el estudio de los sistemas pensionales, con el fin de obtener explicaciones acertadas sobre el impacto de la dinámica poblacional y del mercado laboral en el bienestar de la creciente población de adultos mayores, así como sobre las repercusiones en la sostenibilidad financiera de los sistemas pensionales.

En el presente artículo, por medio del empleo de la técnica del Modelo Basado en Agentes (MBA), se evalúan las interrelaciones entre las características del mercado laboral y la dinámica poblacional que tienen implicaciones en la falta de cobertura y sostenibilidad financiera del sistema pensional colombiano (SPC).

Con este fin, a partir de las características actuales definidas por la normatividad y los usos vigentes, se construye un algoritmo que permite la realización de simulaciones computarizadas de las decisiones individuales y colectivas de los agentes. También, se presenta el desarrollo del MBA propuesto para el estudio multivariado de las dinámicas del mercado laboral y poblacional respecto al sistema pensional, especificando las variables y los detalles de la programación. Para mostrar las posibilidades de la aproximación del modelo, se han analizado tres simulaciones, cada una determinada por las hipótesis que establecen el comportamiento de los agentes dentro del modelo. 
En la primera simulación, se analiza el sistema pensional vigente por dos motivos: el primero es estudiar el efecto de algunos de los parámetros del modelo, así como la participación de las variables poblacionales y del mercado laboral con la menor interferencia ocasionada por efectos exógenos; y el segundo es servir como base para comparar las demás simulaciones realizadas.

En esta primera simulación, se observa la distribución de la población de acuerdo con el "fenómeno de pirámide invertida", que sumado al alto porcentaje de individuos en informalidad que no cotizan al sistema pensional, hace que la financiación del sistema por parte del Estado incremente en cada periodo analizado.

En la segunda simulación, se evalúa el efecto de incrementar las tasas de participación del empleo formal en el sistema pensional, manteniendo las condiciones de la primera simulación. En este punto, se evidencia cómo al intentar corregir el problema de la informalidad sin políticas adecuadas que permitan fortalecer el sistema pensional, se aumenta la responsabilidad del Estado y se hace necesario que su financiación sea mayor.

La tercera simulación se caracteriza por el análisis el sistema pensional en dos pilares, según los cuales todos los individuos con salarios superiores a un salario mínimo aportan a los dos sistemas, de manera que toda cotización por encima de un salario mínimo es enviada al régimen de ahorro individual y todos los cotizantes aportan sobre un salario mínimo al régimen de prima media. En esta simulación, se muestra cómo se frena la aceleración del déficit, pero no se logra aumentar la cobertura; finalmente, se presentan los resultados del ejercicio de simulación, las conclusiones y las recomendaciones derivadas del estudio.

\section{Modelo basado en agentes y simulación del sistema pensional colombiano}

La simulación basada en agentes ha mostrado ser un método útil para modelar sistemas complejos, y muy especialmente sistemas sociales (Conte et al., 1997; Gilbert y Troitzsch, 1999; Gilbert y Terna, 2000) en los que se desea estudiar a profundidad la relación entre las cualidades y los comportamientos de los individuos frente a las características generales del grupo (Gilbert y Troitzsch, 1999; Squazzoni, 2008). Los modelos basados en agentes se distinguen por incorporar varios agentes que son, en mayor o menor grado, autónomos, heterogéneos e independientes; cada uno tiene sus propias metas y objetivos, y en general, son capaces de interaccionar entre ellos y con su entorno (Torsun, 1995).

Actualmente, los MBA han tomado gran importancia en la investigación social. En Colombia, se han desarrollado estudios utilizando la simulación basada en agentes en el 
mercado laboral (Novoa \& Vargas, 2011) y en otras ciencias (Moreno, 2007; Pastrana et al. 2005). Sin embargo, no se han elaborado estudios del SPG utilizando esta metodología. Este sistema se ha analizado previamente en un intento por encontrar las razones de la baja cobertura y financiación (Santa María, 2010), estudios que se limitan a describir la situación del sistema y en algunos casos especifican cifras del mercado laboral y de la dinámica poblacional (Sánchez, 2008), pero no se analiza el impacto de los desequilibrios del mercado laboral, ni los cambios demográficos en el sistema pensional.

La abstracción del sistema pensional muestra las relaciones entre los agentes, las variables y los métodos implicados; mediante simulación basada en agentes, los componentes básicos del sistema real están explícita e individualmente representados en el modelo (Edmonds et al., 2001). Los parámetros utilizados en el modelo obtenidos del estudio previo del sistema pensional, así como el mercado laboral y la dinámica de la población se muestran en el anexo 1.

Así, los límites que establecen los componentes básicos del sistema real corresponden a los que determinan a los agentes del modelo, y las interacciones entre los componentes básicos del sistema real corresponden a las interacciones entre los agentes del modelo (Edmonds et al., 2001; Galán et al., 2009). Esta correspondencia directa contrasta con el tradicional uso de "agentes representativos" y es capaz de aumentar la precisión y el rigor científico de los modelos formales así realizados. En el SPC, interaccionan cinco tipos de agentes, a saber:

E1 Estado: quien hace las veces de legislador y supervisor de las leyes que refieren al SPC; vela por su cumplimiento, regula a los demás agentes que intervienen en el sistema y garantiza la pensión mínima a través del fondo de garantías de la pensión mínima y del presupuesto nacional.

AFP del RAIS: administra los recursos provenientes de los cotizantes y garantiza una rentabilidad mínima de dichos recursos, para lo cual tiene fondos de clasificación del riesgo, en los que el cliente tiene el derecho a elegir el riesgo del portafolio en el cual quiere que se encuentren sus recursos; el porcentaje de garantía lo imparte el Estado a través de la Superintendencia Financiera de Colombia (SFG). Los recursos de los afiliados no se mezclan con el capital de la AFP, con el fin de garantizar su seguridad en todo caso, y el monto de la pensión de los afiliados que cumplan con los requisitos dependerá del valor ahorrado en el fondo, garantizando en todo caso la pensión mínima.

AFP del RPM: administra los recursos provenientes de los cotizantes y garantiza una rentabilidad mínima de dichos recursos, para lo cual tiene fondos de clasificación del riesgo, en los que el cliente tiene el derecho a elegir el riesgo del portafolio en el cual quiere que se encuentren sus recursos; el porcentaje de garantía lo imparte el Estado a través de la SFC. Los recursos de los afiliados no se mezclan con el capital de la AFP, a 
fin de asegurar su seguridad en todo caso, y el monto de la pensión de los afiliados que cumplan con los requisitos dependerá de la base salarial del cotizante de acuerdo con la Ley 100 de 1993.

Ministerio de Trabajo: administra el fondo de subsistencia y el fondo de solidaridad. El primero es un aporte destinado a grupos poblaciones que, por sus características y condiciones, no tienen acceso a los sistemas de seguridad social (por ejemplo, trabajadores independientes urbanos y rurales, desempleados, madres comunitarias, discapacitados, y concejales pertenecientes a municipios de categorías 4, 5 y 6). El segundo, con el programa "Colombia Mayor", busca aumentar la protección a los adultos mayores que se encuentran desamparados, es decir que no cuentan con una pensión o viven en la indigencia o en la extrema pobreza, por medio de la entrega de un subsidio económico. Además, el Ministerio de Trabajo es el encargado de formular, adoptar, dirigir y coordinar las políticas de empleo, trabajo, previsión y seguridad social, dentro de las directrices aprobadas por el Gobierno.

Individuo: toda persona natural que por sus características es sujeto de cobertura del sistema pensional en la vejez, ya sea por las AFP o por el Ministerio de Trabajo. Estas personas en todo tiempo están relacionadas con el sistema pensional, ya sea como cotizantes, pensionados, beneficiarios o como no cotizantes y posibles candidatos a beneficios de los programas del Ministerio de Trabajo. En el submodelo de la dinámica del mercado laboral, se incluyen los agentes ocupados formales, ocupados informales y desocupados. 
Modelación basada en agentes en el sistema pensional colombiano.

Nancy del Carmen Quiceno Cárdenas

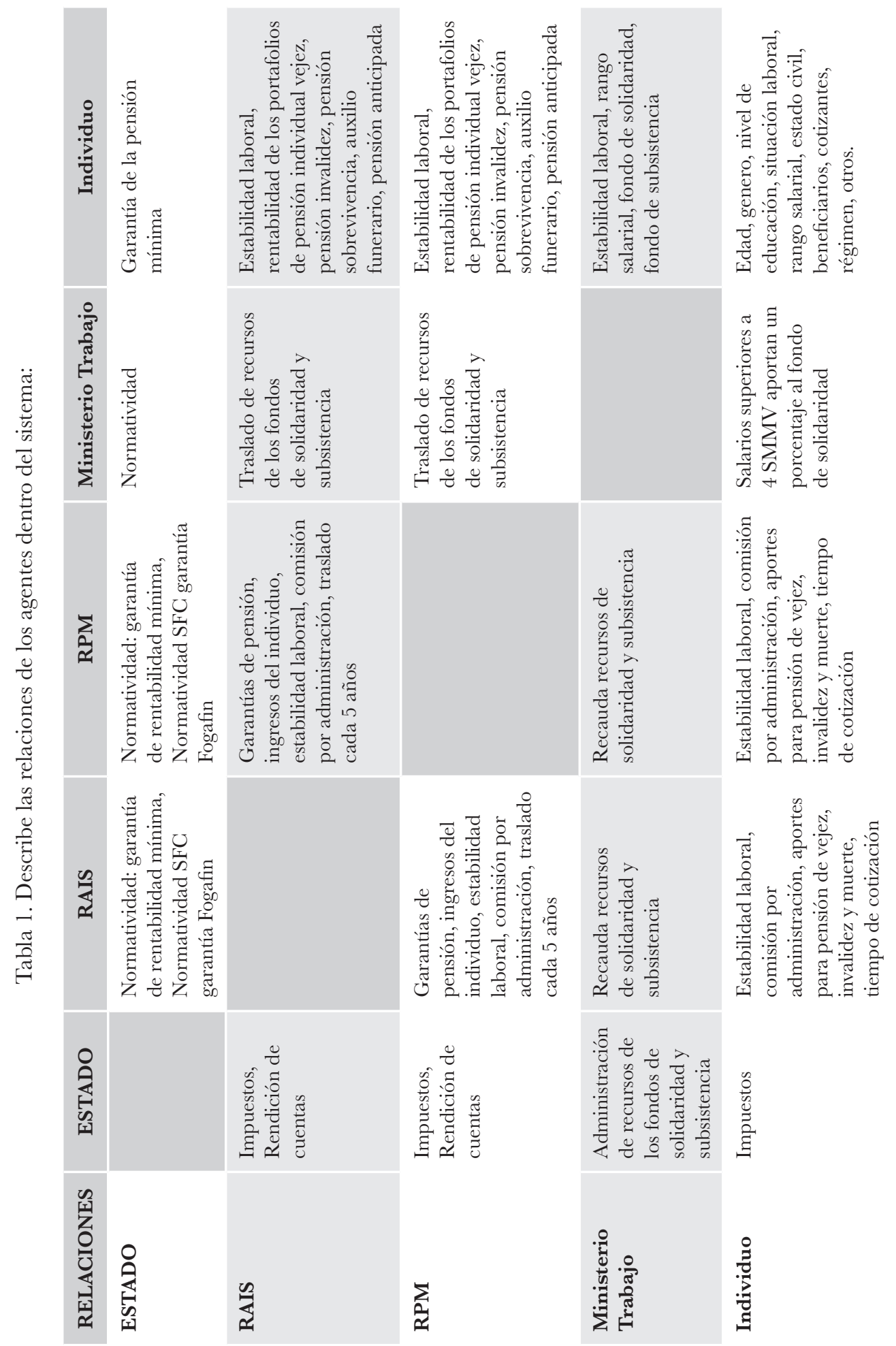


A continuación, se describen las variables y los métodos de los agentes en el estudio del sistema pensional en Colombia:

Tabla 2. El Estado: características y funciones en el modelo.

\section{Estado}

\section{Todas las variables iniciales corresponden a la distribución reportada por el DANE} y la SFG, respectivamente

El análisis se realiza en número de salarios mínimos, por lo cual se omite la proyección del salario mínimo.

RAIS

El crecimiento anual de la proporción de pensionados por el RAIS con un salario mínimo es proyectado con series de tiempo y es calculado para cada quinquenio.

El crecimiento anual de la proporción de cotizantes en cada rango de mesadas es proyectado con series de tiempo y es calculado para cada quinquenio.

El análisis se realiza en número de salarios mínimos, por lo cual se omite la proyección del salario mínimo.

El crecimiento anual de la proporción de pensionados por el RPM con un salario mínimo es proyectado con series de tiempo y es calculado para cada quinquenio.

RPM

El crecimiento anual de la proporción de pensionados en cada rango de mesadas es proyectado con series de tiempo y calculado para cada quinquenio.

El crecimiento anual de la proporción de cotizantes en cada rango de mesadas es proyectado con series de tiempo y es calculado para cada quinquenio.

Para todas las características sociodemográficas del individuo como género y estado civil, entre otras, el crecimiento anual de las proporciones de las categorías de cada variable se calculó utilizando series de tiempo y se proyectó para cada quinquenio.

La probabilidad de sobrevivir al siguiente quinquenio se calculó utilizando las tablas de mortalidad proporcionadas por la SFC, con la respectiva tabla quinquenal en la que se asume distribución uniforme dentro del intervalo.

Individuo

Para todas las variables del mercado laboral como ocupación, desocupación, informalidad, entre otras, el crecimiento anual de las proporciones de las categorías de cada variable se calculó utilizando series de tiempo y se proyectó para cada quinquenio.

El crecimiento de la distribución de los cotizantes y pensionados en cada uno de los regímenes se calculó utilizando series de tiempo y se proyectó para cada quinquenio.

Fuente: elaboración propia. 
Modelación basada en agentes en el sistema pensional colombiano.

Tabla 3. RAIS: características y funciones en el modelo.

\section{RAIS}

Todas las variables iniciales corresponden a la distribución reportada por el DANE y la SFG, respectivamente individuos en cada rango salarial. El crecimiento de la participación de los individuos en cada rango salarial depende las características demográficas y del mercado laboral.

\begin{tabular}{l|l} 
RPM & El crecimiento de la proporción de asociados al RAIS que se cambia al RPM es
\end{tabular} calculado utilizando series de tiempo y es proyectado para cada quinquenio.

El valor de los fondos de subsistencia y solidaridad, la pensión mínima, depende de la concentración de individuos con salarios superiores a 4 SMLV. El crecimiento de la participación de los individuos en cada rango salarial depende de las características

Ministerio demográficas y del mercado laboral.

de Trabajo El número de candidatos a "Colombia Mayor" depende de las características demográficas y del mercado laboral. El crecimiento de la proporción de adultos mayores candidatos a "Colombia Mayor" se calculó utilizando series de tiempo y se proyectó para cada quinquenio.

Individuo

El valor recaudado por el RAIS depende de las variables demográficas y del mercado laboral en Colombia. El crecimiento depende de la relación de las variables.

Fuente: elaboración propia.

Tabla 4. RPM: características y funciones en el modelo.

\section{RPM}

Todas las variables iniciales corresponden a la distribución reportada por el DANE y la SFG, respectivamente

El valor recaudado por el RPM con destino a la bolsa común depende de las variables

Estado demográficas y del mercado laboral en Colombia. El crecimiento depende de la relación de las variables.

RAIS

El crecimiento de la proporción de asociados al RPM que se cambia al RAIS se calculó utilizando series de tiempo y se proyectó para cada quinquenio.

El valor de los fondos de subsistencia y solidaridad depende de la concentración de individuos con salarios superiores a $4 \mathrm{SMLV}$. El crecimiento de la participación de los individuos en cada rango salarial depende de las características demográficas y del

Ministerio mercado laboral.

de Trabajo El número de candidatos a "Colombia Mayor" depende de las características demográficas y del mercado laboral. El crecimiento de la proporción de adultos mayores candidatos a "Colombia Mayor" se calculó utilizando series de tiempo y se proyectó para cada quinquenio.

El valor recaudado por el RPM con destino a la bolsa común depende de las variables

Individuo demográficas y del mercado laboral en Colombia. El crecimiento depende de la relación de las variables.

Fuente: elaboración propia. 
Tabla 5. Ministerio de Trabajo: características y funciones en el modelo.

\begin{tabular}{|c|c|}
\hline \multicolumn{2}{|r|}{ Ministerio de Trabajo } \\
\hline \multicolumn{2}{|c|}{$\begin{array}{l}\text { Todas las variables iniciales corresponden a la distribución reportada por el DANE } \\
\text { y la SFG, respectivamente }\end{array}$} \\
\hline Estado & $\begin{array}{l}\text { El valor de los fondos de subsistencia y solidaridad, la pensión mínima, depende de la } \\
\text { concentración de individuos con salarios superiores a } 4 \text { SMLV. El crecimiento de la } \\
\text { participación de los individuos en cada rango salarial depende de las características } \\
\text { demográficas y del mercado laboral. }\end{array}$ \\
\hline RAIS & $\begin{array}{l}\text { El valor recaudado por el RAIS con destino a los fondos de subsistencia y solidaridad } \\
\text { depende de la concentración de individuos con salarios superiores a } 4 \mathrm{SMLV} \text {. El } \\
\text { crecimiento de la participación de los individuos en cada rango salarial depende de las } \\
\text { características demográficas y del mercado laboral. }\end{array}$ \\
\hline RPM & $\begin{array}{l}\text { El valor recaudado por el RPM con destino a los fondos de subsistencia y solidaridad, } \\
\text { la pensión mínima, depende de la concentración de individuos con salarios superiores } \\
\text { a } 4 \text { SMLV. El crecimiento de la participación de los individuos en cada rango salarial } \\
\text { depende de las características demográficas y del mercado laboral. }\end{array}$ \\
\hline Individuo & $\begin{array}{l}\text { Teniendo en cuenta las características del individuo y suponiendo independencia } \\
\text { estadística entre las variables, el individuo que no tenga opción de pensionarse puede } \\
\text { o no acceder a los beneficios de alguno de los programas del Ministerio de Trabajo. } \\
\text { El crecimiento anual de la proporción de individuos pensionados y no pensionados, } \\
\text { candidatos y no candidatos a "Colombia Mayor" se calculó por series de tiempo y se } \\
\text { proyectó para cada quinquenio. }\end{array}$ \\
\hline
\end{tabular}

Fuente: elaboración propia.

Tabla 6. Individuo: características y funciones en el modelo.

\section{Individuo}

Todas las variables iniciales corresponden a la distribución reportada por el DANE y la $\mathrm{SFC}$, respectivamente

El crecimiento anual de las proporciones de los individuos que cotizan y que no cotizan al RAIS 1 se calculó utilizando series de tiempo y se proyectó para cada quinquenio.

no se pensionan por el RAIS depende de las características sociodemográficas y del no se pensionan por el RAIS depende de las características sociodemográficas y de mercado laboral en Colombia, ya que se deben cumplir los requisitos de tiempo y edad.

El crecimiento anual de las proporciones de los individuos en cada rango salarial sobre el cual se cotiza al RAIS se calculó utilizando series de tiempo y se proyectó para cada quinquenio.

\section{Individuo}




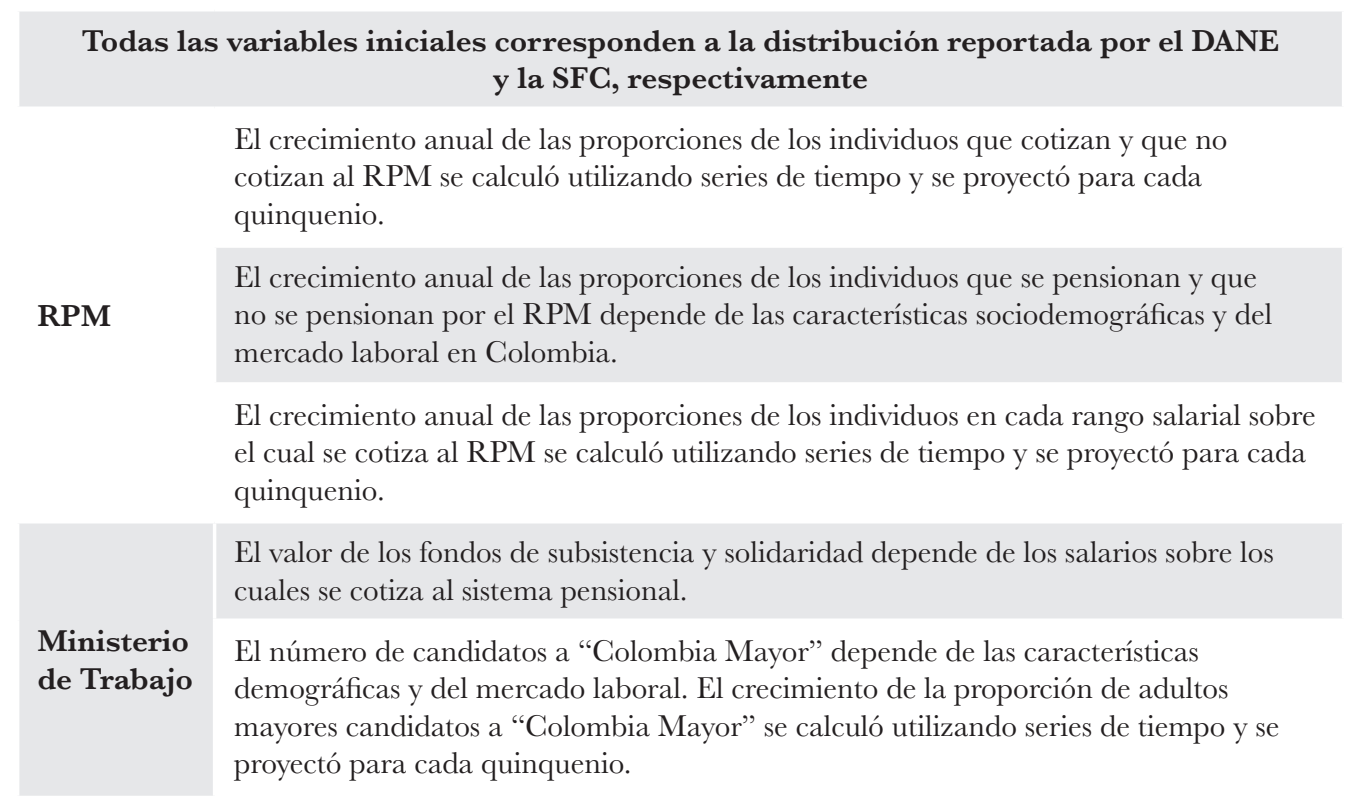

Fuente: elaboracion propia.

En el anexo 1, se observa la diversidad de opciones y elementos que afronta el agente en el modelo para el sistema pensional, el mercado laboral y la dinámica poblacional.

A continuación, se presenta el modelo que se definió para evaluar la cobertura y la sostenibilidad del sistema pensional colombiano. Se trata de una aproximación híbrida compuesta por un submodelo estadístico de caracterización de la cobertura a los individuos del sistema y de su sostenibilidad con un modelo basado en agentes que integra, a su vez, un modelo de dinámica poblacional y de mercado laboral en Colombia.

La influencia de la dinámica poblacional y del mercado laboral en la cobertura y sostenibilidad de los sistemas pensionales han sido comprobados por separado en varias oportunidades (Franco, 2012; Clavijo, 2011; Santa María 2010; Sánchez 2008). Por lo tanto, los modelos que pretenden estudiar estos tópicos de los sistemas pensionales incluyen los submodelos de dinámica poblacional y de mercado laboral.

La dinámica poblacional y del mercado laboral por sí sola implica un problema complejo dada la dependencia de diversos factores, como las políticas públicas y el entorno cultural, que afectan las decisiones de cada uno de los individuos. No obstante, es posible modelarlos con la identificación de sus principales características demográficas y con la adaptabilidad en el tiempo; esta última es medida como la tendencia de las distribuciones de cada una de las características de los individuos en horizontes de tiempo determinados. 
De esta manera, no es posible identificar con exactitud las proporciones futuras de las propiedades de los individuos. Sin embargo, en el modelo es necesario incluir las dinámicas del mercado laboral y de la población, con el fin de identificar su influencia conjunta en el SPC. Para la identificación de las distribuciones iniciales de las características en la población colombiana, se tomó la información del Departamento Administrativo Nacional de Estadística (DANE) y de la Superintendencia Financiera de Colombia (SFC). Los cambios de las distribuciones se estimaron utilizando su respectiva información histórica. Las proporciones iniciales de las características de los individuos y sus tendencias futuras en periodos quinquenales son presentadas en el anexo 1.

La distribución de los individuos, de acuerdo con sus características demográficas, se detalla a partir de la información reportada por el DANE. Dichas características cambian en el tiempo conforme a la adaptabilidad de los individuos a los factores antes mencionados. Para la toma de decisiones de los agentes, no solo se denotan las características económicas sino también las de un código cultural individual que define las posibilidades de comportamiento con respecto a la cotización al sistema pensional. Para ello, se utiliza el genotipo individual de cada agente como un vector multidimensional, en el que varios de sus valores pueden cambiar durante las iteraciones individuales y globales.

Formalmente, la identidad de un individuo A se describe con un vector de dimensión booleano:

$$
V_{A}=\left(v_{A, 1}, v_{A, 2}, v_{A, 3}, \ldots, v_{A, n}\right) \operatorname{conv}_{A, i} \in\{0,1\} ; i=1,2, \ldots, n
$$

Es importante notar que, con la definición anterior, se tendrían $2^{n}$ identidades diferentes para cada individuo, donde $V_{A}$ y $V_{B}$ son identidades diferentes si por lo menos difieren en un componente. La característica $V_{A, n+1}$ del individuo depende de las con $V_{A, I}$ con i | $\mathrm{i}=1,2, \ldots \mathrm{n}, V_{A, n+1}$. indica si el individuo está cubierto por el sistema pensional o no, ya sea por pensión propia, familiar o por el consorcio "Colombia Mayor".

La cobertura en la cual se hace énfasis en este documento es la del adulto mayor, por lo cual, para el cálculo de la cobertura del sistema, se tomaron los $V_{A, n+1}$ con pensión de vejez, los $V_{A, n+1}$ con pensión de invalidez o sobrevivencia con edad superior a los 60 años, y los adultos mayores cubiertos con "Colombia Mayor".

Tal que la cobertura total del sistema está dada por:

$$
\operatorname{Cob}_{T}=\frac{\sum v_{A, n+1}}{\sum v_{A, j}}
$$


Modelación basada en agentes en el sistema pensional colombiano.

Nancy del Carmen Quiceno Cárdenas

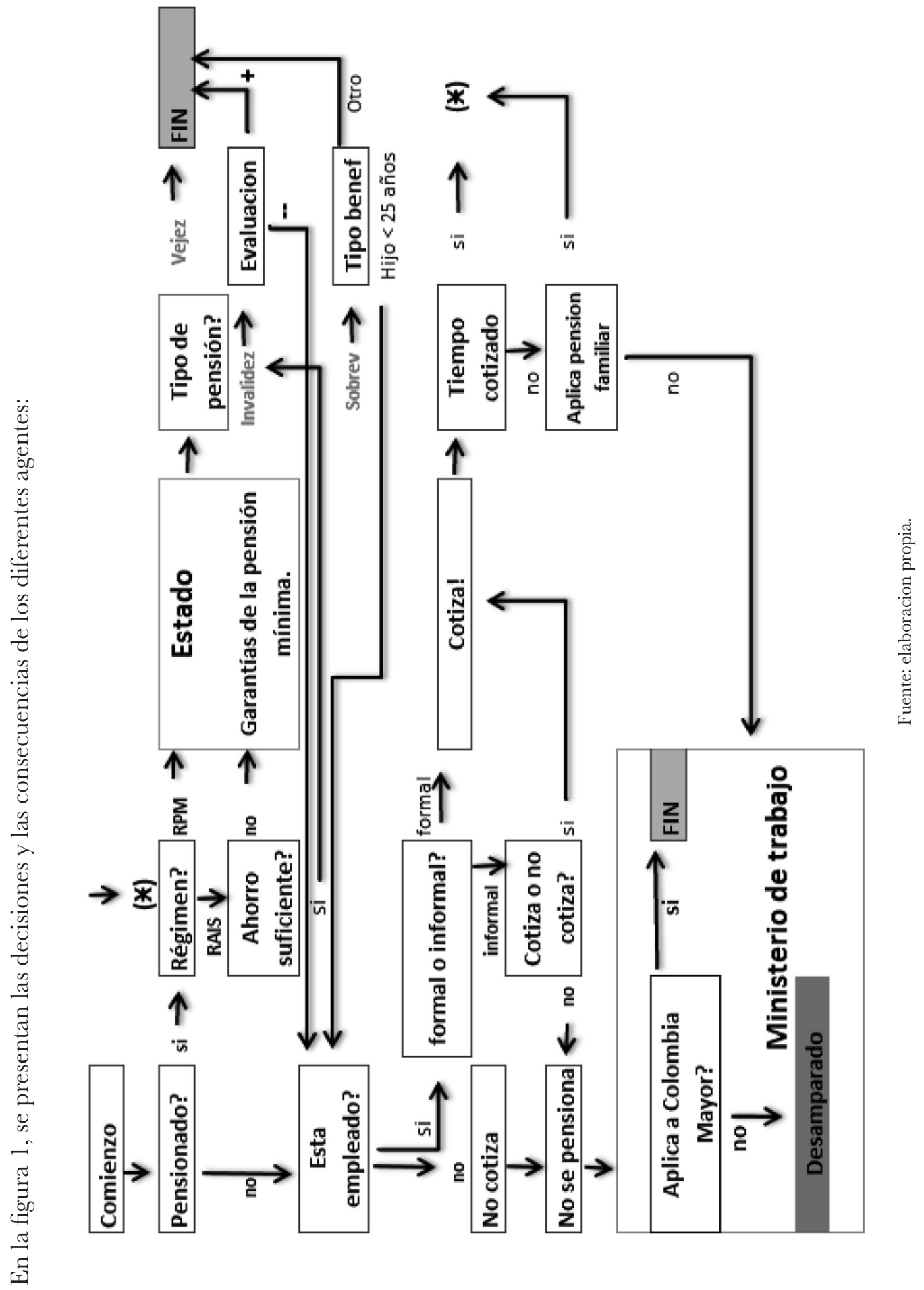


Siendo $V_{A, j}$ la característica del individuo que indica si tiene edad superior a 60 años. $\mathrm{Y} \sum v_{A, \mathrm{n}+1}$ está dada por :

$$
\sum v_{A, n+1}=\sum v_{A, n_{1}}+\sum v_{A, n_{2}}+\sum v_{A, n_{3}}
$$

Con:

$v_{A, \mathrm{n}_{1}}$ : Característica del individuo que indica si es pensionado por vejez.

$v_{A, n_{2}}$ : Característica del individuo que indica si es pensionado por invalidez o sobrevivencia y edad superior a 60 años.

$v_{A, n_{3}}$ : Característica del individuo cubierto por "Colombia Mayor".

Por lo tanto, es evidente que se puede discriminar cada cobertura de manera individual y que la cobertura total se obtiene a partir de las coberturas individuales.

La sostenibilidad del sistema está dada por:

Con:

$$
\operatorname{Sos}_{T}=\frac{\sum M_{v_{A, m}} v_{A, m} v_{A, n+1}}{\sum S_{v_{A, s}} v_{A, s} v_{A, k}}
$$

$v_{A, m}$ : Característica del individuo que indica si el valor de la mesada está en el rango m.

$M_{v_{A, m}}:$ Marca de clase del intervalo m.

$v_{A, \mathrm{~K}}$ : Característica del individuo que indica si cotiza al sistema pensional.

$v_{A, \mathrm{~S}}$ : Característica del individuo que indica si el valor del salario del individuo está en el rango $\mathrm{S}$.

$S_{v_{A, \mathrm{~S}}}:$ Marca de clase del intervalo S.

Donde:

$$
\sum M_{v_{A, m}} v_{A, m} v_{A, n+1}
$$

incluye las mesadas de los pensionados por vejez y la cobertura de "Colombia Mayor".

$$
\sum S_{v_{A, S}} v_{A, s} v_{A, k}
$$

incluye los aportes a pensión y los aportes a los fondos de solidaridad, subsistencia y garantías. 
La sostenibilidad extendida del sistema está dada por:

$$
\operatorname{Sos}_{T_{\text {Exte }}}=\frac{\sum M_{v_{A, m}} v_{A, m} v_{A, n+1}}{\sum S_{v_{A, s}} v_{A, s} v_{A, k}}
$$

Con : $v_{A, m}$ : Característica del individuo que indica si el valor de la mesada está en el rango $\mathrm{m}$.

$M_{v_{A, m}}:$ Marca de clase del intervalo $\mathrm{m}$.

$v_{A, \mathrm{~K}}$ : Característica del individuo que indica si cotiza al sistema pensional.

$v_{A, \mathrm{~S}}$ : Característica del individuo que indica si el valor del salario del individuo está en el rango $\mathrm{S}$.

$S_{v_{A, \mathrm{~S}}}:$ Marca de clase del intervalo S.

Donde:

$$
\sum M_{v_{A, m}} v_{A, m} v_{A, n+1}
$$

incluye las mesadas de los pensionados por vejez, invalidez, sobrevivencia y la cobertura de "Colombia Mayor".

$$
\sum S_{v_{A, s}} v_{A, s} v_{A, k}
$$

incluye los aportes a pensión y los aportes a los fondos de solidaridad, subsistencia y garantías.

$\sum M_{V_{A, m}} V_{A, m} V_{A, n+1}=\sum M_{v_{A, m}} v_{A, m} v_{A, n+1}, \sum M_{v_{A, m}} v_{A, m} v_{A, n+1}, \sum M_{v_{A, m}} v_{A, m} v_{A, n+1}\left(v_{A, r m p}, v_{A, r a i s}, v_{A, \text { ColMy }}\right)^{T}$

Se tiene que:

$$
=\sum M_{V_{A, m}} V_{A, m} V_{A, n+1} V+\sum M_{v_{A, m}} v_{A, m} v_{A, n+1} v_{A, \text { rais }}+\sum M_{v_{A, m}} v_{A, m} v_{A, n+1} v_{A, C o l M y}
$$

$v_{A, 1}$ : Característica del individuo que indica si recibe la mesada del RPM,

del RAIS o de "Colombia Mayor". $l \in\{r m p$, rais, ColMy $\}$

$\sum S_{v_{A, s}} v_{A, s} v_{A, k}=\sum S_{v_{A, s}} v_{A, s} v_{A, k}, \sum S_{v_{A, s}} v_{A, s} v_{A, k} v_{A, \text { rais }}, \sum S_{v_{A, s}} v_{A, s} v_{A, k}\left(v_{A, \text { rmp }}, v_{A, \text { rais }}, v_{A, \text { Fondos }}\right)^{T}$

$=\sum S_{v_{A, s}} v_{A, S} v_{A, k} V_{A, r V m}+\sum S_{v_{A, s}} v_{A, s} v_{A, k} v_{A, \text { rais }}+\sum S_{v_{A, s}} v_{A, S} v_{A, k} v_{A, \text { Fondos }}$

$v_{A, \mathrm{q}}$ : Característica del individuo que indica si cotiza al RPM o al RAIS y el aporte a los fondos. $q \in\{$ rmp, rais, ColMy $\}$ 
Por lo tanto, es evidente que se puede discriminar la sostenibilidad de cada régimen de manera individual, y que la sostenibilidad total se obtiene a partir de las anteriores y del consorcio "Colombia Mayor".

\section{Dinámica del sistema pensional}

De lo anterior se evidencia que siempre que el valor de las cotizaciones exceda el valor de las mesadas, se tendrá una sostenibilidad directa; sin embargo, esto solo aplica en el régimen de prima media (RPM), en el que los aportes se acumulan en una bolsa común. En el régimen de ahorro individual (RAIS), cada sujeto tiene una cuenta en la que acumula sus aportes para su propia pensión; de modo que la sostenibilidad de las mesadas es responsabilidad de cada cuenta particular, siempre y cuando el monto ahorrado sea suficiente para solventar una pensión de por lo menos un salario mínimo mensual durante el tiempo de pensión; de lo contrario, el fondo de garantías de la pensión mínima y el Estado lo garantizan.

Uno de los grandes problemas es el mercado laboral, en el que no se ha conseguido que el $100 \%$ de las personas interesadas en trabajar y devengar un salario lo consigan, y otro es la tendencia a los salarios cada vez más cercanos al salario mínimo. Es importante recordar que los aportes o las cotizaciones al sistema pensional se hacen tomando como base el salario devengado y que el $100 \%$ de las cotizaciones inferiores a dos salarios mínimos no consiguen ahorrar lo suficiente para solventar su propia pensión. Así mismo, hay que tener presente el trabajo informal o no reglamentado, dado que solo el $20 \%$ de los empleados informales aporta al sistema pensional.

Otro gran problema es la dinámica de población, denotada por las características $p_{A, i}$ que identifican la identidad de cada individuo. Estas características cambian con el tiempo, e incluso determinan si un sujeto sobrevive o no al siguiente periodo de análisis. Si el sujeto es pensionado sin sobrevivientes y fallece antes de comenzar el siguiente periodo, el sistema aumenta su sostenibilidad pero disminuye su cobertura, mientras que si el sujeto es un cotizante sin beneficiarios, el sistema disminuye su sostenibilidad y su cobertura a corto plazo permanece invariante; si tiene beneficiarios, disminuye su sostenibilidad y aumenta su cobertura. Así, todos los individuos interactuan dentro del sistema.

$\operatorname{Tmr}_{i} P_{i, t}=P_{i, 0}\left(1+r_{i}\right)^{T}$ para cada $\mathrm{i}=1,2, \ldots \mathrm{n} \mathrm{El}$ algoritmo sigue un proceso en etapas. En la etapa inicial, se le otorga una identidad a cada agente de un conjunto $\mathrm{F}$ de agentes; esta identidad es determinada a partir de la elección aleatoria de cada uno de los componentes del vector $\mathrm{V}_{\mathrm{A}}$. Luego, se seleccionan los candidatos a pensionarse, y se da la opción a cada uno de los agentes que cotizan al sistema. Este proceso se traduce en una probabilidad $\mathrm{P}$ que depende de la variables que describen la dinámica de población y el mercado laboral, caracterizadas en el vector $\mathrm{V}_{\mathrm{A}}$. Esta probabilidad es sensible al 
tiempo y se supone una dependencia exponencial con la probabilidad $P_{0}$ constante e independiente del agente. $P_{0}$ es un parámetro del modelo y es un vector multidimensional, donde cada $P_{i, 0}$ es el parámetro del modelo en el componente.

Donde $r_{i}$ es la tasa de crecimiento del componente $i$ del vector $P, r_{i}$ se supone constante en el tiempo e independiente del agente. $r_{i}$ es un parámetro del modelo y es el $i$-ésimo componente del vector $r_{i}$. Esta tasa de crecimiento se da anualmente, por lo cual para los siguientes periodos se estimará la tasa.

$P_{-}(\mathrm{i}, 0) \mathrm{ti}=1,2, \ldots \mathrm{n}$

Con $T$ el número de subperiodos en $i=1,2, \ldots, n$ :.

$$
r_{i, T}=\left(1+r_{i, 0}\right)^{T}-1
$$

Si el agente no está pensionado, se calcula suprobabilidad de pensionarse, así decida,según su estado laboral, si cotiza o no al sistema. Si el agente no se encuentra ocupado, su decisión será obligada a no cotizar, y si el agente se encuentra ocupado formalmente, será obligado a cotizar, de modo quela elección se centra en los agentes ocupados informales.

$$
P_{i, t}=P_{i, 0}\left(1+r_{i}\right)^{T}
$$

La segunda etapa del algoritmo se encarga de estimar la cobertura y la sostenibilidad de cada uno de los subsistemas (régimen de ahorro individual, régimen de prima media, “Colombia Mayor").En la identidad del agente, se incluye el subsistema al cual es candidato a pertenecer, por lo quese selecciona aleatoriamente un valor de la mesada. Este valor está determinado por la variable aleatoria,que se distribuye de acuerdo con la función de probabilidad del componente $\mathrm{X}$ del vector $\mathrm{P}$ y varía en el tiempo de acuerdo con el componente del vector m. El proceso está acotado por el tamaño de la población en el t periodo. En cada iteración, el orden de los agentes dentro del conjunto F es aleatorio para evitar posibles desviaciones. 


\section{CĆl'F́= 24}

ISSN: 0124-3551 / Año 16, No 24 / enero-junio / pp. 119-162

La figura 2 muestra el diagrama de flujo del algoritmo:

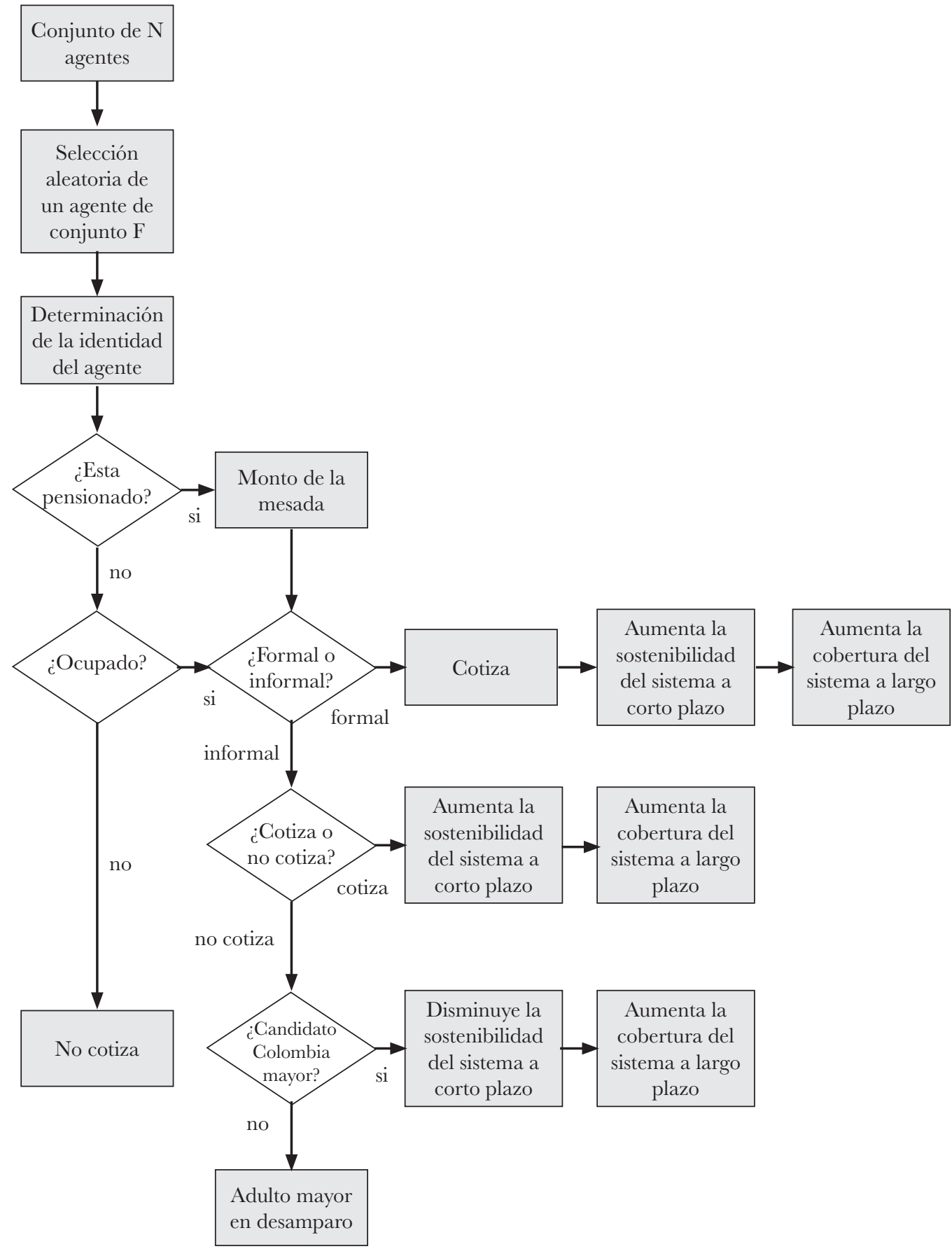

Fuente: elaboracion propia. 


\section{Detalles de la programación}

El modelo para el sistema pensional se lleva a cabo en un número definido de iteraciones. Cada iteración representa la dinámica del sistema pensional, del mercado laboral y de la población al final del periodo analizado, y en cada una se desarrollan ocho procesos, siguiendo el orden que aparece a continuación:

Paso 1: En el modelo del sistema pensional, los agentes pueden ser pensionados o no pensionados.

Paso 2: En el submodelo del mercado laboral, los agentes no pensionados pueden ser ocupados o no ocupados.

Paso 3: Si el agente es no ocupado, se incluye en la lista de candidatos al programa de subsidios del Estado.

Paso 4: En el submodelo del mercado laboral, los agentes ocupados pueden ser formales o informales.

Paso 5: En el modelo del sistema pensional utilizado y en el resultado del submodelo del mercado laboral, el agente puede ser un informal que cotiza o un informal que no cotiza para pensión.

Paso 6: En el modelo del sistema pensional utilizado y en el resultado del submodelo del mercado laboral, el agente que cotiza puede cotizar al RAIS o al RPM.

Paso 7: En el modelo del sistema pensional, el individuo puede ser pensionado por el RAIS o por el RPM.

Paso 8: En el modelo del sistema pensional, con base en la dinámica poblacional y en las características del individuo, se identifica si este es candidato a financiación del Estado o no y el monto de dicha financiación.

En el anexo 1, se puede observar la diversidad de opciones y elementos que afronta el agente en el modelo para el sistema pensional, el mercado laboral y la dinámica poblacional.

\section{Configuración de las simulaciones}

El modelo está particularizado en la evolución de los agentes de los últimos veinte años. $\mathrm{Al}$ ser un modelo complejo, con gran cantidad de parámetros (especificados en el anexo 1), hay dificultad (en términos prácticos) para analizarlo completamente y en todas las combinaciones de parámetros. En todo caso, el diseño del modelo no es su objetivo, sino proporcionar una herramienta que permita entender aspectos sobre la estructura global del sistema, mediante la integración de los subsistemas. 
Para la configuración inicial del sistema, se utilizaron diferentes hipótesis con respecto a la población, los algoritmos y las bases de datos. En cuanto a las simulaciones, existe un problema de escala. Si se intenta simular en forma explícita los cerca de 48 millones de individuos como agentes del modelo, más los diferentes regímenes y opciones de cobertura del sistema, la mayoría de los ordenadores tienen problema de memoria para realizar las simulaciones. Por este motivo, se ha reducido la escala del problema disminuyendo el número de individuos, con un margen de error de la proporción de pensionados $P$, con el fin de $d$ mantener las distribuciones calculadas y estimadas del modelo, y con riesgo $\propto=99.8 \%$ de que el error real supere a, es decir:

Suponiendo un muestreo aleatorio simple, se tiene que:

$$
\begin{gathered}
\operatorname{Pr}(|p-P| \geq d)=a \\
\sigma^{2}=\frac{(N-n) P(1-p)}{(N-1) n}
\end{gathered}
$$

Con $\mathrm{P}$ igual a la probabilidad de pensionarse. Por lo tanto, la fórmula que relaciona a $n$ con el grado de precisión deseado es:

$$
d=t \sqrt{\frac{(N-n) P(1-p)}{(N-1) n}}
$$

Con $p$ igual al valor de la distribución en las colas, y $t$ como el valor de la distribución para este grado de significancia. Al resolver para $n$, se tiene:

$$
n=\frac{\frac{t^{2} P(1-p)}{d^{2}}}{1+\frac{\left(t^{2} P(1-p)-d^{2}\right)}{N d^{2}}}
$$

Como $\mathrm{N}$ es el tamaño de la población, el denominador de la expresión anterior tiende a cero, por lo cual se tiene que

$$
n=\frac{t^{2} P(1-p)}{d^{2}}
$$

Y con los parámetros escogidos, se tendría que:

$$
n=211854
$$


Donde se obtiene que el mínimo tamaño de la muestra para la investigación sea de 211.854 individuos; para el análisis, se incluyen 250.000 habitantes.

Otro aspecto que se plantea es determinar la representación temporal de la simulación, el valor de paso y el horizonte temporal que se quiere simular. El valor de paso está dado por la información disponible en las bases insumo del modelo. Como la mayoría de información es quinquenal, no tiene sentido incluir un valor de paso menor.

En lo concerniente al horizonte temporal, se tomaron espacios de tiempo de 5 años para estar acorde con el valor de paso y se hicieron cálculos para 5, 10 y 15 años. Los resultados con tiempos mayores pierden validez de forma importante, puesto que en horizontes temporales mayores se pueden producir cambios en la dinámica poblacional y en el mercado laboral que ocasionen que cada simulación pierda significado. En cada ejercicio de simulación, se han ejecutado 100 simulaciones, con el fin de reducir el efecto estocástico que tiene el proceso en el cálculo de la media de las distribuciones.

$\mathrm{Al}$ incluir información quinquenal en todas las variables del modelo, se partió del supuesto de distribución uniforme en el interior de cada intervalo en cada subcategoría, por lo cual en el desarrollo del modelo la información de cada variable en cada intervalo de tiempo se centra en la marca de clase.

Para mostrar las posibilidades de la aproximación, se analizaron tres simulaciones, cada una determinada por las hipótesis sobre el comportamiento de los agentes dentro del modelo. La simulación 1 tiene como objetivo establecer la situación del sistema pensional reglamentado por la Ley 100 y sus modificaciones hasta el 31 de diciembre de 2012. La simulación 2 evalúa el efecto de incrementar la tasa de participación del empleo formal en el sistema, manteniendo las condiciones del sistema pensional de la simulación 1. Y la simulación 3 analiza el sistema en dos pilares, en los cuales todos los individuos con salarios superiores a un salario mínimo aportan a los dos sistemas, de manera que toda cotización por encima de un salario mínimo es enviada al régimen de ahorro individual y todos los cotizantes aportan sobre un salario mínimo al régimen de prima media. 


\section{Presentación y análisis de resultados}

\subsection{Simulación 1}

Esta simulación presenta un análisis del sistema pensional colombiano reglamentado por la Ley 100 de 1993 y sus modificaciones. Su estudio se justifica básicamente por dos razones: la primera es analizar el sistema pensional vigente estudiando el efecto de algunos de los parámetros del modelo, así como la participación de las variables poblacionales y del mercado laboral con la menor interferencia ocasionada por efectos exógenos; la segunda es servir de base para comparar las demás simulaciones realizadas.

Esta simulación se caracteriza por la configuración de la dinámica poblacional y del mercado laboral sin intervención. No se incluye ningún parámetro de disonancia, ni mecanismos que interfieran en el modelo de la dinámica del sistema pensional. En el anexo 1, se muestran los parámetros iniciales utilizados en el modelo.

A continuación, se muestra la dinámica del mercado laboral tras finalizar el primer, el segundo, el tercer y el cuarto quinquenio de análisis con 100 simulaciones.

El resultado del modelo es en cuanto a la relación de los individuos ocupados, desocupados e inactivos para cada uno de los periodos. Se evidencia un incremento en el porcentaje de desempleados e inactivos, mientras que el número de ocupados disminuye en cada quinquenio.

Tabla 7. Dinámica del mercado laboral. Simulación 1.

\begin{tabular}{|l|c|c|c|c|}
\hline & Q1 & Q2 & Q3 & Q4 \\
\hline Desocupados & \multicolumn{3}{|c|}{ Media de los resultados } \\
\hline Ocupados & $12.21 \%$ & $12.69 \%$ & $13.26 \%$ & $13.88 \%$ \\
\hline Inactivos & $51.80 \%$ & $50.27 \%$ & $49.18 \%$ & $48.51 \%$ \\
\hline
\end{tabular}

Fuente: elaboración propia.

La tabla 7 muestra la media de los resultados de la primera simulación. Se evidencia una creciente tasa de desempleo al final de cada quinquenio, lo que la sitúa al final del cuarto quinquenio en el 13,88 \%, así como una decreciente tasa de ocupados que al final de cuarto quinquenio se encuentra en el 48,51 \% comparado con el 51,80 \% al final del primer quinquenio, teniendo en cuenta una dinámica poblacional con el fenómeno de pirámide invertida.

En cuanto al nivel de informalidad, los resultados del modelo, en lo concerniente a la relación entre el empleo formal y el informal, muestra un incremento en el empleo informal en cada uno de los periodos analizados. 
Tabla 8. Dinámica del mercado laboral. Simulación 1.

\begin{tabular}{|c|c|c|c|c|}
\hline & Q1 & Q2 & Q3 & Q4 \\
\hline \multicolumn{5}{|c|}{ Media de los resultados } \\
\hline Ocupados_Informales & $56.29 \%$ & $54.72 \%$ & $53.18 \%$ & $50.79 \%$ \\
\hline Ocupados_Formales & $43.71 \%$ & $45.28 \%$ & $46.82 \%$ & $49.21 \%$ \\
\hline
\end{tabular}

La tabla 8 muestra una caída de la media del nivel de empleo informal, y dado que este valor está construido con base en el total de ocupados, el empleo formal presenta aumento, situándose en el 49,21\% al final del cuarto quinquenio.

A continuación, se muestran los resultados del modelo en cuanto a la dinámica poblacional tras finalizar el primer, el segundo, el tercer y el cuarto quinquenio de análisis con 100 simulaciones.

Se incrementa el porcentaje de adultos mayores y los individuos en edad de trabajar (de 12 a 60 años), mientras que el porcentaje de menores de 12 años muestra descenso en cada periodo. Lo anterior evidencia el fenómeno poblacional de pirámide invertida. La tabla 9 muestra la media de las distribuciones de la población y su evolución en los quinquenios analizados.

Tabla 9. Dinámica poblacional. Simulación 1.

\begin{tabular}{|c|c|c|c|c|}
\hline & Q1 & Q2 & Q3 & Q4 \\
\hline \multicolumn{5}{|c|}{ Media de los resultados } \\
\hline Adultos mayores & $7.94 \%$ & $8.58 \%$ & $9.00 \%$ & $9.50 \%$ \\
\hline Edad entre 12-60 años & $65.00 \%$ & $66.11 \%$ & $66.69 \%$ & $68.17 \%$ \\
\hline Menores de 12 años & $27.06 \%$ & $25.31 \%$ & $24.04 \%$ & $22.34 \%$ \\
\hline
\end{tabular}

Estos son los resultados del modelo en lo que respecta al sistema pensional colombiano, del primer al cuarto quinquenio de análisis con 100 simulaciones.

Los resultados del modelo en lo concerniente a la distribución de aportantes y pensionados en cada uno de los regímenes evidencia un incremento en el porcentaje de pensionados en el RAIS, mientras en el RPM el porcentaje presenta disminución. Igualmente, el porcentaje de cotizantes al RPM disminuye y en el del RAIS aumenta en cada periodo de análisis. La tabla 10 muestra la evolución de la media de las simulaciones en cada quinquenio. 
Tabla 10. Dinámica del sistema pensional. Simulación 1.

\begin{tabular}{|l|c|c|c|c|}
\hline & Q1 & Q2 & Q3 & Q4 \\
\hline Cotizantes RPM & \multicolumn{3}{|c|}{ Media de los resultados } \\
\hline Cotizantes RAIS & $25.14 \%$ & $23.68 \%$ & $22.12 \%$ & $20.79 \%$ \\
\hline Pensionados RPM & $74.86 \%$ & $76.32 \%$ & $77.88 \%$ & $79.21 \%$ \\
\hline Pensionados RAIS & $54.18 \%$ & $92.32 \%$ & $91.71 \%$ & $88.28 \%$ \\
\hline
\end{tabular}

Fuente: elaboración propia.

En cuanto a cobertura del sistema pensional, a continuación se presenta el resultado de la primera simulación:

El modelo muestra la relación entre el monto total de las mesadas pagadas por cada uno de los regímenes y los respectivos montos de cotización en cada uno. Ambos regímenes aumentan sus montos de pago de mesadas y aportes recibidos. Sin embargo, la velocidad del incremento en el monto de los aportes es, por mucho, mayor en el RAIS que en el RPM; por el contrario, en el monto de las mesadas el incremento es mayor en el RPM que en el RAIS. La tabla 11 presenta la evolución de la media de los aportes y las mesadas en cada régimen en millones de SMLV.

Tabla 11. Dinámica del sistema pensional. Simulación 1.

\begin{tabular}{|l|c|c|c|c|}
\hline & Q1 & Q2 & Q3 & Q4 \\
\hline Vlr_mesadas_rais & 37.97 & Media de los resultados & & 60.59 \\
\hline Vlr_mesadas_rpm & 58.51 & 42.32 & 49.52 & 217.35 \\
\hline Vlr_cotiz_rpm & 18.91 & 102.40 & 156.29 & 40.06 \\
\hline Vlr_cotiz_rais & 209.24 & 26.81 & 33.54 & 291.45 \\
\hline
\end{tabular}

Fuente: elaboración propia.

En cuanto a los adultos que no logran pensionarse, a continuación se presenta el resultado de la simulación.

Los resultados del modelo muestran el incremento en el fondo de garantías de la pensión mínima, ya que este fondo no ha presentado movimientos de salidas de dinero hacia los pensionados; el modelo recoge la falta de información al respecto y solo refleja las entradas de dinero.

El fondo de solidaridad mantiene un capital constante, puesto que así se destinan los beneficios del programa para el adulto mayor, y los pensionados con altos montos hacen aportes a este fondo. Mientras tanto, el fondo de subsistencia disminuye hasta anularse 
en el cuarto quinquenio, debido especialmente a la falta de contracción de salarios de altos montos. De acuerdo con el modelo, los aportantes para este periodo no tendrán salarios superiores a 16 SMLV y habrá alta concentración en los salarios de hasta 4 SMLV.

La tabla 12 muestra la evolución de la media del valor de los fondos de subsistencia, solidaridad y garantías de la pensión mínima en millones de SMLV, y la evolución de la media de candidatos a "Colombia Mayor", antes de la evaluación para hacerlos beneficiarios del sistema en millones de individuos.

Tabla 12. Dinámica del sistema pensional. Simulación 1.

\begin{tabular}{|c|c|c|c|c|}
\hline & Q1 & Q2 & Q3 & $Q^{4}$ \\
\hline \multicolumn{5}{|c|}{ Media de los resultados } \\
\hline vbenef_colom_mayor & 2.71 & 3.19 & 4.13 & 4.56 \\
\hline vfondo_subsit & 0.23 & 0.21 & 0.00 & - \\
\hline vfondo_solidaridad & 0.15 & 0.14 & 0.09 & 0.07 \\
\hline vfondo_gar_pen_min & 10.46 & 13.84 & 15.80 & 15.97 \\
\hline
\end{tabular}

Fuente: elaboración propia

La tabla 13 muestra la media de las distribuciones de los índices señalados antes. Se evidencia disminución en la cobertura con y sin "Colombia Mayor", teniendo en cuenta el fenómeno de pirámide invertida que indica que el número de adultos mayores sobre el cual está construido el índice está en aumento. Por el contrario, el índice de sostenibilidad presenta aumento, lo cual muestra la falta de sostenibilidad del sistema en todos los quinquenios estudiados, incluyendo las pensiones de sobrevivencia e invalidez (índice extendido) y excluyendo a estas últimas en el índice total no extendido.

Tabla 13. Índices del sistema pensional. Simulación 1.

\begin{tabular}{|c|c|c|c|c|}
\hline & Q1 & Q2 & Q3 & Q4 \\
\hline \multicolumn{5}{|c|}{ Media de los resultados } \\
\hline Cobertura Total & $31 \%$ & $27 \%$ & $22 \%$ & $22 \%$ \\
\hline Cobertura sin Colombia Mayor & $22 \%$ & $22 \%$ & $20 \%$ & $18 \%$ \\
\hline Sostenibilidad Total Extendida & 1.97 & 2.50 & 3.16 & 3.58 \\
\hline Sostenibilidad Total & 1.57 & 2.00 & 2.53 & 3.08 \\
\hline
\end{tabular}

Fuente: elaboración propia 


\subsection{Simulación 2}

En la segunda simulación, se evalúa el efecto de incrementar las tasas de participación del empleo formal en el sistema, manteniendo las condiciones del sistema pensional de la primera simulación.

Esta simulación se caracteriza por la misma configuración de la primera, pero añade un factor multiplicativo a la tasa de crecimiento de la participación del empleo formal; es claro que el crecimiento de las tasas de desempleo y de informalidad disminuye. Los parámetros del mercado laboral son tomados del total de la población con el factor de crecimiento en el subperiodo.

Tabla 14. Factor de crecimiento empleo.

\begin{tabular}{|c|c|}
\hline Variable & Factor Crecimiento \\
\hline Desempleo & -0.05 \\
\hline Ocupacion Formal & -0.04 \\
\hline Ocupacion Informal & 0.09 \\
\hline
\end{tabular}

Fuente: elaboración propia

A continuación, se muestra la dinámica del mercado laboral tras finalizar el primero, el segundo, el tercer y el cuarto quinquenio de análisis con 100 simulaciones.

Dado que el factor multiplicativo afecta la distribución de la ocupación formal e informal, pero no directamente la desocupación o la inactividad, el modelo muestra la relación de los individuos ocupados, desocupados e inactivos para cada uno de los periodos. Se evidencian resultados similares a los de la primera simulación, es decir, se incrementó el porcentaje de desempleados e inactivos, mientras que el número de ocupados disminuyó en cada quinquenio.

Tabla 15. Dinámica del mercado laboral. Simulación 2.

\begin{tabular}{|c|c|c|c|c|}
\hline & Q1 & Q2 & Q3 & $Q^{4}$ \\
\hline \multicolumn{5}{|c|}{ Media de los resultados } \\
\hline Desocupados & $12.15 \%$ & $12.71 \%$ & $13.40 \%$ & $13.81 \%$ \\
\hline Ocupados & $51.81 \%$ & $50.26 \%$ & $49.52 \%$ & $48.78 \%$ \\
\hline Inactivos & $36.56 \%$ & $36.94 \%$ & $37.47 \%$ & $38.07 \%$ \\
\hline
\end{tabular}

Fuente: elaboración propia.

La tabla 15 describe la media de los resultados de la segunda simulación. Se muestra la creciente tasa de desempleo al final de cada quinquenio, que se sitúa en el 13,81\% 
al final del cuarto quinquenio, y la decreciente tasa de ocupados que al final de cuarto quinquenio se encuentra en el 48,78\%, comparado con el 51,81\% al final del primer quinquenio. Lo anterior tiene en cuenta una dinámica poblacional con el fenómeno de pirámide invertida.

En cuanto al nivel de informalidad, de acuerdo con las condiciones del ambiente diseñado, los resultados del modelo en lo concerniente a la relación entre el empleo formal y el informal muestran un incremento en el empleo formal en cada uno de los periodos analizados.

Tabla 16. Dinámica del mercado laboral. Simulación 2.

\begin{tabular}{|c|c|c|c|c|}
\hline & Q1 & Q2 & Q3 & $Q^{4}$ \\
\hline \multicolumn{5}{|c|}{ Media de los resultados } \\
\hline Ocupados_Informales & $54.23 \%$ & $50.97 \%$ & $46.71 \%$ & $42.66 \%$ \\
\hline Ocupados_Formales & $45.77 \%$ & $49.03 \%$ & $53.29 \%$ & $57.34 \%$ \\
\hline
\end{tabular}

Fuente: elaboración propia.

De lo anterior, debido a que se incluye el factor multiplicativo, en la tabla 16 se evidencia un aumento progresivo en el nivel de empleo formal, y dado que este valor está construido con base en el total de ocupados, el empleo informal presenta disminución y se sitúa en el 42,66\% al final del cuarto quinquenio.

La dinámica poblacional no se modificó en el diseño de la segunda simulación, por lo cual el resultado es análogo a la primera simulación, tras finalizar el primero, el segundo, el tercer y el cuarto quinquenio de análisis con 100 simulaciones.

Análogo a la primera simulación, el modelo muestra un incremento en el porcentaje de adultos mayores e individuos en edad de trabajar (de 12 a 60 años), mientras que el porcentaje de menores de 12 años muestra un descenso en cada periodo. Lo anterior evidencia el fenómeno poblacional de pirámide invertida. La tabla 17 muestra la media de las distribuciones de la población y su evolución en los quinquenios analizados.

Tabla 17. Dinámica poblacional. Simulación 2.

\begin{tabular}{|c|c|c|c|c|}
\hline & Q1 & Q2 & Q3 & $\mathrm{Q}^{4}$ \\
\hline \multicolumn{5}{|c|}{ Media de los resultados } \\
\hline Adultos mayores & $7.95 \%$ & $8.38 \%$ & $9.00 \%$ & $9.33 \%$ \\
\hline Edad entre 12-60 años & $64.90 \%$ & $65.96 \%$ & $66.91 \%$ & $68.12 \%$ \\
\hline Menores de 12 años & $27.15 \%$ & $25.67 \%$ & $24.08 \%$ & $22.54 \%$ \\
\hline
\end{tabular}


A continuación, se describen los resultados del modelo en lo que respecta al sistema pensional colombiano, del primer al cuarto quinquenio de análisis con 100 simulaciones.

De acuerdo con el diseño de la segunda simulación, si bien el porcentaje de cotizantes aumenta a razón del porcentaje de empleados formales, la relación de cotizantes en los dos regímenes no se ve afectada de manera representativa. Los resultados del modelo evidencian un incremento en el porcentaje de pensionados en el RAIS. El porcentaje de cotizantes al RPM disminuye y al RAIS aumenta en cada periodo de análisis.

La tabla 18 muestra la evolución de la media de las simulaciones en cada quinquenio.

Tabla 18. Dinámica poblacional. Simulación 2.

\begin{tabular}{|l|c|c|c|c|}
\hline & Q1 & Q2 & Q3 & Q4 \\
\hline & \multicolumn{5}{c|}{ Media de los resultados } & & \\
\hline Cotizantes RPM & $27.00 \%$ & $23.61 \%$ & $22.10 \%$ & $20.87 \%$ \\
\hline Cotizantes RAIS & $73.00 \%$ & $76.39 \%$ & $77.90 \%$ & $79.13 \%$ \\
\hline Pensionados RPM & $93.95 \%$ & $93.50 \%$ & $92.23 \%$ & $92.03 \%$ \\
\hline Pensionados RAIS & $6.05 \%$ & $6.50 \%$ & $7.77 \%$ & $7.97 \%$ \\
\hline
\end{tabular}

Fuente: elaboración propia.

A continuación, se muestra el resultado de la segunda simulación. El modelo muestra la relación entre el monto total de las mesadas pagadas por cada uno de los regímenes y los respectivos montos de cotización en cada uno. Ambos regímenes aumentan sus montos de pago de mesadas y aportes recibidos; sin embargo, la velocidad del incremento en el monto de los aportes es, por mucho, mayor en el RAIS que el RPM. Por el contrario, en el monto de las mesadas el incremento es mayor en el RPM que en el RAIS.

En relación con la simulación 1, en ambos regímenes se presenta mayor aumento en el valor cotizado, mientras que el valor de las mesadas pagadas solo aumenta en el último quinquenio. Esto es debido a que el incremento de la formalidad comienza en el periodo uno y el tiempo mínimo cotizado actual en el sistema pensional colombiano es de 1300 semanas.

La tabla 19 presenta la evolución de la media de los aportes y las mesadas en cada régimen en millones de SMLV. 
Tabla 19. Dinámica del sistema pensional. Simulación 2.

\begin{tabular}{|l|c|c|c|c|}
\hline & Q1 & Q2 & Q3 & Q4 \\
\hline & \multicolumn{4}{c|}{ Media de los resultados } \\
Vlr_mesadas_rais & $38.49 \%$ & $47.05 \%$ & $58.60 \%$ & $71.88 \%$ \\
\hline Vlr_mesadas_rpm & $50.05 \%$ & $112.36 \%$ & $174.38 \%$ & $240.22 \%$ \\
\hline Vlr_cotiz_rpm & $19.29 \%$ & $27.95 \%$ & $34.52 \%$ & $41.72 \%$ \\
\hline Vlr_cotiz_rais & $186.51 \%$ & $244.74 \%$ & $269.47 \%$ & $296.99 \%$ \\
\hline
\end{tabular}

Fuente: elaboración propia.

Con respecto a los adultos que no logran pensionarse, a continuación se presenta el resultado de la simulación.

De manera análoga a la simulación 1, hay un incremento en el fondo de garantías de la pensión mínima, ya que este fondo no ha presentado movimientos de salidas de dinero hacia los pensionados, y el modelo recoge la falta de información al respecto y solo refleja las entradas de dinero. El fondo de solidaridad disminuye su capital dado que de allí se destinan los beneficios del programa para el adulto mayor; gran parte de los aportes a este fondo los realizan los pensionados con altos montos. Esto ocurre mientras que el fondo de subsistencia disminuye hasta anularse en el cuarto quinquenio, debido especialmente a la falta de contracción de salarios de altos montos; de acuerdo con el modelo, los aportantes para este periodo no tendrán salarios superiores a 16 SMLV y habrá alta concentración en los salarios de hasta 4 SMLV.

La tabla 20 muestra la evolución de la media del valor de los fondos de subsistencia, solidaridad y garantías de la pensión mínima en millones de SMLV, así como la evolución de la media de candidatos a "Colombia Mayor" antes de la evaluación para hacerlos beneficiarios del sistema, en millones de individuos.

Tabla 20. Dinámica del sistema pensional. Simulación 2.

\begin{tabular}{|c|c|c|c|c|}
\hline & Q1 & Q2 & Q3 & $Q^{4}$ \\
\hline \multicolumn{5}{|c|}{ Media de los resultados } \\
\hline vbenef_colom_mayor & $2.71 \%$ & $2.93 \%$ & $3.07 \%$ & $3.56 \%$ \\
\hline vfondo_subsit & $0.23 \%$ & $0.21 \%$ & $0.00 \%$ & - \\
\hline vfondo_solidaridad & $0.15 \%$ & $0.12 \%$ & $0.09 \%$ & $0.05 \%$ \\
\hline vfondo_gar_pen_min & $10.46 \%$ & $13.85 \%$ & $17.29 \%$ & $17.71 \%$ \\
\hline
\end{tabular}

Fuente: elaboración propia. 
La tabla 21 muestra la media de las distribuciones de los índices señalados antes. Se evidencia aumento en la cobertura con y sin "Colombia Mayor", teniendo en cuenta el fenómeno de pirámide invertida, que indica que el número de adultos mayores sobre el cual está construido el índice está en aumento. De igual manera, el índice de sostenibilidad presenta aumento, lo cual muestra la falta de sostenibilidad del sistema en todos los quinquenios estudiados, incluyendo las pensiones de sobrevivencia e invalidez (índice extendido) y excluyendo estas últimas en el índice total no extendido; también, los índices asociados a la sostenibilidad del sistema son mayores a los de la primera simulación.

Tabla 21. Índices sistema pensional. Simulación 2.

\begin{tabular}{|l|c|c|c|c|}
\hline & Q1 & Q2 & Q3 & Q4 \\
\hline & Media de los resultados & & $36.0 \%$ \\
\hline Cobertura Total & $28.2 \%$ & $28.9 \%$ & $30.7 \%$ & $26.9 \%$ \\
\hline Cobertura sin Colombia Mayor & $22.7 \%$ & $24.3 \%$ & $24.7 \%$ & 3.82 \\
\hline Sostenibilidad Total Extendida & 1.97 & 2.67 & 3.36 & 3.05 \\
\hline Sostenibilidad Total & 1.57 & 2.13 & 2.69 & \\
\hline
\end{tabular}

Fuente: elaboración propia.

\subsection{Simulación 3}

La tercera simulación se caracteriza por el análisis del sistema pensional en dos pilares, en los que todos los individuos con salarios superiores a un salario mínimo aportan a ambos sistemas, de manera que toda cotización por encima de un salario mínimo es enviada al régimen de ahorro individual y todos los cotizantes aportan sobre un salario mínimo al régimen de prima media.

Esta simulación tiene la misma configuración de la primera, pero con el añadido de un factor de cotización único para el régimen de prima media (RPM), calculado en el $16 \%$ del SMLV, y un factor de cotización variable para el régimen de ahorro individual (RAIS), calculado en el $16 \%$ del monto que sobrepasa un salario mínimo en valor del salario de cada individuo. El RPM otorgaría el mismo subsidio a todas las pensiones y el RAIS otorgaría una pensión de acuerdo con el monto ahorrado. De esta manera, se anula el parámetro de elección del régimen al cual se desea ahorrar.

A continuación, se muestra la dinámica del mercado laboral al finalizar el primero, el segundo, el tercer y el cuarto quinquenio de análisis con 100 simulaciones.

Dado que la definición del escenario no afecta directamente a la desocupación ni a la inactividad, el modelo muestra la relación de los individuos ocupados, desocupados e inactivos para cada uno de los periodos. Aquí, se evidencian resultados similares a los 
de la primera simulación, es decir, el incremento en el porcentaje de desempleados e inactivos, mientras el número de ocupados disminuye en cada quinquenio.

Tabla 22. Dinámica del mercado laboral. Simulación 3.

\begin{tabular}{|l|c|c|c|c|}
\hline & Q1 & Q2 & Q3 & Q4 \\
\hline \multicolumn{4}{|c|}{ Media de los resultados } \\
\hline Desocupados & \multicolumn{2}{|c|}{ los } & & \\
\hline Ocupados & $12.21 \%$ & $12.79 \%$ & $13.25 \%$ & $13.84 \%$ \\
\hline Inactivos & $51.74 \%$ & $50.37 \%$ & $49.28 \%$ & $48.06 \%$ \\
\hline
\end{tabular}

Fuente: elaboración propia.

La tabla 22 muestra la media de los resultados de la primera simulación. Se muestra la creciente tasa de desempleo al final de cada quinquenio, la cual se sitúa al final del cuarto quinquenio en el $13,84 \%$, y la decreciente tasa de ocupados que al final del cuarto quinquenio se encuentra en el 48,06 \% comparado con el 51,74\% al final del primer quinquenio, en una dinámica poblacional con el fenómeno de pirámide invertida.

De manera análoga, el nivel de informalidad, de acuerdo con el modelo, muestra incremento en el empleo informal en cada uno de los periodos analizados.

Tabla 23. Dinámica del mercado laboral. Simulación 3.

\begin{tabular}{|l|c|c|c|c|}
\hline & Q1 & Q2 & Q3 & Q4 \\
\hline & \multicolumn{1}{c|}{ Media de los resultados } & & \\
\hline Ocupados_Informales & $56.33 \%$ & $54.79 \%$ & $52.06 \%$ & $49.85 \%$ \\
\hline Ocupados_Formales & $43.67 \%$ & $45.21 \%$ & $47.94 \%$ & $50.15 \%$ \\
\hline
\end{tabular}

Fuente: elaboración propia.

La tabla 23 muestra una caída del nivel de empleo informal, y como este valor está construido con base en el total de ocupados, el empleo formal presenta aumento y se sitúa en el $50,15 \%$ al final del cuarto quinquenio.

A continuación, se muestran los resultados del modelo en dinámica poblacional al finalizar el primero, el segundo, el tercer y el cuarto quinquenio de análisis con 100 simulaciones.

El modelo muestra el incremento en el porcentaje de adultos mayores y en individuos en edad de trabajar (edad de 12 a 60 años), mientras el porcentaje de menores de 12 años muestra descenso en cada periodo. Lo anterior es evidencia del fenómeno poblacional 
de pirámide invertida. La tabla 24 muestra la media de las distribuciones de la población y su evolución en los quinquenios analizados.

Tabla 24. Dinámica poblacional. Simulación 3.

\begin{tabular}{|l|c|c|c|c|}
\hline & Q1 & Q2 & Q3 & Q4 \\
\hline \multicolumn{4}{|c|}{ Media de los resultados } \\
\hline Adultos mayores & $7.97 \%$ & $8.40 \%$ & $8.82 \%$ & $9.46 \%$ \\
\hline Edad entre 12-60 años & $65.02 \%$ & $66.12 \%$ & $66.96 \%$ & $68.13 \%$ \\
\hline Menores de 12 años & $27.01 \%$ & $25.47 \%$ & $24.22 \%$ & $22.42 \%$ \\
\hline
\end{tabular}

Fuente: elaboración propia.

A continuación, se presentan los resultados del modelo en lo respectivo al sistema pensional colombiano. Del primer al cuarto quinquenio de análisis con 100 simulaciones.

En los resultados del modelo en lo concerniente a la distribución de aportantes y pensionados en cada uno de los regímenes, dado que todo individuo que se pensiona por el RAIS también está pensionado por el RPM, pero no todos los individuos pensionados por el RPM están pensionados por el RAIS, se evidencia mayor concentración en los pensionados por el RPM. En los aportantes, todos los cotizantes aportan al RPM, por lo cual este es igual al $100 \%$ de los cotizantes, mientras los cotizantes al RAIS disminuyen a razón de los salarios. La tabla 25 muestra la evolución de la media de las simulaciones en cada quinquenio.

Tabla 25. Dinámica poblacional. Simulación 3.

\begin{tabular}{|l|c|c|c|c|}
\hline & Q1 & Q2 & Q3 & Q4 \\
\hline Cotizantes RPM & \multicolumn{2}{|c|}{ Media de los resultados } & & $100.00 \%$ \\
\hline Cotizantes RAIS & $100.00 \%$ & $100.00 \%$ & $100.00 \%$ & $29.53 \%$ \\
\hline Pensionados RPM & $51.86 \%$ & $47.47 \%$ & $39.46 \%$ & $96.24 \%$ \\
\hline Pensionados RAIS & $94.57 \%$ & $95.05 \%$ & $95.37 \%$ & $3.76 \%$ \\
\hline
\end{tabular}

Fuente: elaboración propia.

A continuación, se muestra el resultado de la tercera simulación. El modelo describe la relación entre el monto total de las mesadas pagadas por cada uno de los regímenes y los respectivos montos de cotización en cada uno. Ambos regímenes aumentan sus montos de pago de mesadas y aportes recibidos. Sin embargo, en esta simulación la velocidad del incremento en el monto de los aportes es, por mucho, mayor en el RAIS en que en el RPM; al igual que el monto de las mesadas, en el que el incremento es mayor en el RAIS 
que en el RPM. En relación con la simulación uno, el valor de las mesadas pagadas por el RPM no aumenta aceleradamente; por el contrario, aumenta con una velocidad baja. Esto es debido a que el RPM otorgó un subsidio igual a todos los pensionados.

Tabla 26. Dinámica del sistema pensional. Simulación 3.

\begin{tabular}{|l|c|c|c|c|}
\hline & Q1 & Q2 & Q3 & Q4 \\
\hline \multicolumn{7}{r}{} & \multicolumn{2}{c|}{ Media de los resultados } & & \\
\hline Vlr_mesadas_rais & $46.50 \%$ & $61.36 \%$ & $79.64 \%$ & $102.07 \%$ \\
\hline Vlr_mesadas_rpm & $34.52 \%$ & $41.98 \%$ & $48.59 \%$ & $55.29 \%$ \\
\hline Vlr_cotiz_rpm & $10.08 \%$ & $10.97 \%$ & $12.00 \%$ & $13.05 \%$ \\
\hline Vlr_cotiz_rais & $210.30 \%$ & $222.77 \%$ & $245.94 \%$ & $268.69 \%$
\end{tabular}

Fuente: elaboración propia.

En cuanto a los adultos que no logran pensionarse bajo el supuesto de que el fondo de garantías de la pensión mínima sigue vigente, de manera análoga a la simulación uno, hay un incremento en el fondo de garantías de la pensión mínima, dado que este fondo no ha presentado moviente de salidas de dinero hacia los pensionados, y el modelo recoge la falta de información al respecto y solo refleja las entradas de dinero.

El fondo de solidaridad disminuye su capital puesto que de allí se destinan los beneficios del programa para el adulto mayor; gran parte de los aportes a este fondo los realizan los pensionados con altos montos. Esto ocurre mientras que el fondo de subsistencia disminuye hasta anularse en el cuarto quinquenio, debido especialmente a la falta de contracción de salarios de altos montos. De acuerdo con el modelo, los aportantes para este periodo no tendrán salarios superiores a 16 SMLV y habrá alta concentración en los salarios de hasta 4 SMLV.

La tabla 27 muestra la evolución de la media del valor de los fondos de subsistencia, solidaridad y garantías de la pensión mínima en millones de SMLV, y la evolución de la media de candidatos a "Colombia Mayor" antes de la evaluación para hacerlos beneficiarios del sistema, en millones de individuos.

Tabla 27. Dinámica del sistema pensional. Simulación 3.

\begin{tabular}{|l|c|c|c|c|}
\hline & Q1 & Q2 & Q3 & Q4 \\
\hline vbenef_colom_mayor & \multicolumn{2}{|c|}{ Media de los resultados } & & $4.59 \%$ \\
\hline vfondo_subsit & $2.71 \%$ & $3.17 \%$ & $4.16 \%$ & - \\
\hline vfondo_solidaridad & $0.23 \%$ & $0.21 \%$ & $0.00 \%$ & $0.09 \%$ \\
\hline vfondo_gar_pen_min & $0.15 \%$ & $0.17 \%$ & $17.74 \%$ & $21.36 \%$ \\
\hline
\end{tabular}


La tabla 28 muestra la media de las distribuciones de los índices señalados antes. Se evidencia disminución en la cobertura con y sin "Colombia Mayor", teniendo en cuenta el fenómeno de pirámide invertida, que indica que el número de adultos mayores sobre el cual está construido el índice está en aumento. Contrariamente, el índice de sostenibilidad presenta estancamiento, lo cual muestra la estabilidad del sistema en todos los quinquenios estudiados, incluyendo las pensiones de sobrevivencia e invalidez (índice extendido) y excluyendo a estas últimas en el índice total no extendido.

Tabla 28. Índices del sistema pensional. Simulación 3.

\begin{tabular}{|l|c|c|c|c|}
\hline & Q1 & Q2 & Q3 & Q4 \\
\hline & Media de los resultados & & $21 \%$ \\
\hline Cobertura Total & $33 \%$ & $25 \%$ & $23 \%$ & $18 \%$ \\
\hline Cobertura sin Colombia Mayor & $19 \%$ & $21 \%$ & $20 \%$ & 1.50 \\
\hline Sostenibilidad Total Extendida & 1.60 & 1.51 & 1.51 & 1.20 \\
\hline Sostenibilidad Total & 1.28 & 1.21 & & \\
\hline
\end{tabular}

Las tres simulaciones evidencian distribución poblacional relacionada con el fenómeno de pirámide invertida, es decir, se incrementa la concentración de adultos mayores mientras la de menores de 12 años disminuye, lo que a largo plazo ocasiona mayor monto pagado por mesadas a los pensionados, junto con la desaceleración en el monto cotizado.

En la simulación 3, este fenómeno no afectó en gran medida al RPM, dado que este otorga el mismo beneficio a todos los pensionados. Sin embargo, no soluciona el problema de desfinanciamiento, pues si bien los montos de las pensiones son menores, también lo son los montos aportados por los cotizantes. Así mismo, en los tres ambientes se evidencia alta concentración en los salarios bajos, especialmente en el tercer y el cuarto quinquenio, lo cual causa que las mesadas en estos periodos, incluso para la tercera simulación, se concentren en el RPM.

En lo concerniente a cobertura, la simulación 1 evidencia desprotección del adulto mayor, con aceleración desde el segundo periodo. En la simulación 2, si bien se incrementa el porcentaje de trabajadores formales y se aumenta la cobertura con disminución del número de adultos mayores candidatos a ser beneficiarios de "Colombia Mayor", el incremento en el porcentaje de salarios y mesadas por debajo de 4 SMLV ocasiona mayor participación del Estado para su financiación.

Este último efecto es causa de que el fondo de garantías de la pensión mínima casi desaparezca desde el tercer periodo analizado, puesto que dicho fondo se financia con las pensiones superiores a $4 \mathrm{SMLV}$, y al concentrarse los salarios en estos montos, no hay aportes al fondo; de igual manera sucede con el fondo de subsistencia. El fondo de solidaridad también se financia con los salarios altos, por lo cual disminuye su valor, aunque 
logra permanecer vigente gracias a los aportes de los pensionados que logran altas pensiones; es decir, aun cuando los salarios tienden a disminuir, en el cuarto quinquenio se mantienen pensiones altas gracias a los aportes realizados desde años anteriores al primer quinquenio e incluso durante el primer quinquenio analizado.

\section{Conclusiones}

Subsanar el problema del déficit fiscal con la promoción de la formalización del mercado laboral pero con bajos salarios, o reducir el desempleo con la integración de mano de obra poco calificada al mercado laboral, son medidas que a corto plazo permitirían a las AFP contar con un capital adicional para fortalecer sus inversiones provenientes de los aportes de nuevos cotizantes. No obstante, medidas de este tipo no garantizan la sostenibilidad futura del sistema, en tanto que contribuyen a incrementar el déficit fiscal gubernamental, debido a la mayor participación de individuos de rangos salariales bajos como principales beneficiarios futuros de pensión.

Todas las simulaciones del modelo desarrollado en este trabajo incluyen a la dinámica poblacional colombiana, caracterizada por la participación creciente de adultos mayores, por la disminución de la población joven menor de 12 años y por la alta presencia de la informalidad laboral de la población económicamente activa (PEA) que cotiza al sistema pensional, todo lo cual arroja como resultado la necesidad de incrementar la financiación del sistema con recursos públicos.

De manera específica, mediante la segunda simulación, en la que se evalúa el efecto de incrementar las tasas de participación del empleo formal en el sistema manteniendo las condiciones actuales del sistema pensional, se pone en evidencia cómo al intentar corregir el problema de la informalidad sin políticas laborales adecuadas que contribuyan al fortalecimiento de la productividad laboral y mediante la promoción de empleos de bajos salarios, no se garantiza el sostenimiento de los fondos de garantías de la pensión mínima, de subsistencia y de solidaridad, y hace necesaria una mayor responsabilidad del Estado.

Por su parte, en la tercera simulación, caracterizada por el análisis del sistema pensional en dos pilares en los que todos los individuos con salarios superiores a un salario mínimo aportan a los dos sistemas, si bien se frena la aceleración del déficit, no se logra aumentar la cobertura. Lo anterior, junto con el fenómeno de incremento de la participación de individuos con bajos salarios, ocasionaría que los fondos de garantías de pensión mínima, subsistencia y solidaridad, desaparecieran casi por completo dejando en total desamparo a los adultos mayores que no logren acceder a una pensión.

De lo anterior se concluye que sin políticas adecuadas concernientes al mercado laboral y al sistema pensional en Colombia, los intentos por solucionar los problemas de cobertura y financiamiento del sistema convergen en la solución de uno de ellos y en la 
profundización del otro; de esta manera, el intento por disminuir el déficit fiscal agudiza la baja cobertura, y al intentar aumentar la cobertura, se acentúa el déficit fiscal.

Como conclusión adicional, los resultados de las tres simulaciones contempladas en el modelo basado en agentes en este trabajo son consistentes con los resultados de los trabajos de Santa María (2010) y de Sánchez (2008), en la perspectiva teórica institucionalista y estructuralista. Mediante el modelado basado en agentes y las correspondientes simulaciones, se logra una aproximación a la dinámica del sistema pensional que contempla, al mismo tiempo, la informalidad del mercado laboral y la dinámica poblacional colombiana, en un horizonte temporal de veinte años.

Se propone complementar este análisis con un escenario adicional que involucre las condiciones de la simulación 2, en la cual se evalúa el efecto de incrementar las tasas de participación del empleo formal en el sistema manteniendo las condiciones actuales del sistema pensional, y las de la simulación 3, en la que se analiza el sistema pensional en dos pilares con el fin de obtener una visión más amplia de la problemática y sus soluciones.

\section{Recomendaciones}

Una medida que se puede implementar para disminuir el déficit es mejorar los aportes que se realizan a los fondos de solidaridad, garantías de la pensión mínima y subsistencia, los cuales en la actualidad tienden a desaparecer e incrementan las obligaciones del Estado en materia de financiación del sistema pensional. El aumento en el aporte a la solidaridad afectaría a los salarios altos, mientras que se beneficiarían los individuos con bajo nivel de ingreso, siempre que cuenten con las semanas necesarias para gozar de este privilegio.

Si bien esta medida mejora el recaudo en las cuentas de ahorro previsional, disminuye el déficit general en el momento de retiro y aumenta el valor en los fondos de garantías de la pensión mínima, subsistencia y solidaridad. Sin embargo, esto representaría un costo adicional para las empresas y el Estado, por lo cual se hace indispensable construir políticas estatales a largo plazo para mejorar la productividad de los trabajadores de rango salarial más bajo (salarios inferiores a los 4 SMLV), en quienes se concentra el mercado laboral colombiano.

Para aumentar la cobertura, se deben estudiar detalladamente los efectos directos e indirectos que pueden originar el cambio en una política pensional sobre la economía, la sociedad y las AFP. Como se concluyó en este trabajo, más personas aportando al sistema pensional no implica mayor sostenibilidad; aumentar el número de personas cotizando con una dinámica poblacional con modelo de pirámide invertida no es necesariamente la mejor política para disminuir el déficit fiscal, debido a que no se incrementan los montos en las cuentas individuales, lo que pone en riesgo incluso las pensiones de los que logren cumplir con los requisitos de edad y tiempo. 
Modelación basada en agentes en el sistema pensional colombiano.

\section{Anexos}

\section{Anexos 1}

\begin{tabular}{|c|c|c|c|}
\hline \multicolumn{4}{|c|}{ Parámetros de participación: } \\
\hline Nombre de la variable & Variacion $(\mathbf{r})$ & Nombre de la variable & Variacion $(\mathbf{r})$ \\
\hline crecimiento_poblacion & $-0.2431 \%$ & escolar_0-1 & $-0.1314 \%$ \\
\hline hombres & $-0.0022 \%$ & escolar_1-5 & $-0.7757 \%$ \\
\hline mujeres & $0.0022 \%$ & escolar_6-10 & $0.3440 \%$ \\
\hline soltero & $0.3090 \%$ & escolar_11 & $0.2051 \%$ \\
\hline casado & $-0.1870 \%$ & escolar_12+ & $0.3580 \%$ \\
\hline union_libre & $-0.0802 \%$ & rango_sal_men_2_smlv & $0.3511 \%$ \\
\hline viudo & $-0.0246 \%$ & rango_sal_men_2-4_smlv & $-0.2731 \%$ \\
\hline separ_divorciado & $-0.0150 \%$ & rango_sal_men_4-7_smlv & $-0.0728 \%$ \\
\hline pensionado & $0.0081 \%$ & rango_sal_men_7-10_smlv & $-0.0294 \%$ \\
\hline no_pensionado & $-0.0081 \%$ & rango_sal_men_10-13_smlv & $-0.0095 \%$ \\
\hline pensionado_rpm & $-0.4100 \%$ & rango_sal_men_13-16_smlv & $0.0010 \%$ \\
\hline pensionado_rais & $0.4100 \%$ & rango_sal_men_mas_16_smlv & $0.0327 \%$ \\
\hline pension_vejez & $0.2641 \%$ & cotiza & $0.9183 \%$ \\
\hline pension_invalidez & $0.0408 \%$ & no_cotiza & $-0.9138 \%$ \\
\hline pension_sobrev & $-0.3049 \%$ & cotiza_rpm & $-1.2903 \%$ \\
\hline pens_sobrev_hijoestud & $-0.0001 \%$ & cotiza_rais & $1.2903 \%$ \\
\hline pens_sobrv_otro & $1.7900 \%$ & cambio_regimen & $-1.1672 \%$ \\
\hline edad_entre_0-4 & $0.0349 \%$ & no_cambio_regimen & $1.1672 \%$ \\
\hline edad_entre_5-9 & $0.0560 \%$ & pensine & $0.0081 \%$ \\
\hline edad_entre_10-14 & $0.0229 \%$ & no_pensione & $-0.0081 \%$ \\
\hline edad_entre_15-19 & $-0.0576 \%$ & valor_pens_sobrv_men_2_smlv & $-0.3402 \%$ \\
\hline edad_entre_20-24 & $-0.0710 \%$ & $\begin{array}{l}\text { valor_pens_sobrv_men_2- } \\
\text { 4_smlv }\end{array}$ & $0.2143 \%$ \\
\hline edad_entre_25-29 & $-0.0026 \%$ & $\begin{array}{l}\text { valor_pens_sobrv_men_4- } \\
7 \text { _smlv }\end{array}$ & $0.0853 \%$ \\
\hline edad_entre_30-34 & $0.0105 \%$ & $\begin{array}{l}\text { valor_pens_sobrv_men_7- } \\
\text { 10_smlv }\end{array}$ & $0.0219 \%$ \\
\hline edad_entre_35-39 & $0.0766 \%$ & $\begin{array}{l}\text { valor_pens_sobrv_men_10- } \\
\text { 13_smlv }\end{array}$ & $0.0127 \%$ \\
\hline
\end{tabular}




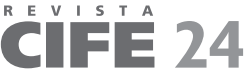

ISSN: 0124-3551 / Año 16, No 24 / enero-junio / pp. 119-162

\begin{tabular}{|c|c|c|c|}
\hline Nombre de la variable & Variacion $(\mathbf{r})$ & Nombre de la variable & $\operatorname{Variacion}(\mathbf{r})$ \\
\hline edad_entre_40-44 & $0.0324 \%$ & $\begin{array}{l}\text { valor_pens_sobrv_men_13- } \\
\text { 16_smlv }\end{array}$ & $0.0066 \%$ \\
\hline edad_entre_45-49 & $-0.0988 \%$ & $\begin{array}{l}\text { valor_pens_sobrv_men_ } \\
\text { mas_16_smlv }\end{array}$ & $-0.0006 \%$ \\
\hline edad_entre_50-54 & $-0.0661 \%$ & $\begin{array}{l}\text { valor_pens_invalid_men_2_ } \\
\text { smlv }\end{array}$ & $0.1186 \%$ \\
\hline edad_entre_55-59 & $-0.0008 \%$ & $\begin{array}{l}\text { valor_pens_invalid_men_2- } \\
\text { 4_smlv }\end{array}$ & $-0.0959 \%$ \\
\hline edad_entre_60-64 & $0.0102 \%$ & $\begin{array}{l}\text { valor_pens_invalid_men_4- } \\
\text { 7_smlv }\end{array}$ & $-0.0252 \%$ \\
\hline edad_entre_65-69 & $0.0340 \%$ & $\begin{array}{l}\text { valor_pens_invalid_men_7- } \\
\text { 10_smlv }\end{array}$ & $0.0025 \%$ \\
\hline edad_entre_70-74 & $0.0208 \%$ & $\begin{array}{l}\text { valor_pens_invalid_men_10- } \\
\text { 13_smlv }\end{array}$ & $-0.0006 \%$ \\
\hline edad_entre_75-79 & $-0.0012 \%$ & $\begin{array}{l}\text { valor_pens_invalid_men_13- } \\
\text { 16_smlv }\end{array}$ & $-0.0004 \%$ \\
\hline edad_entre_80_mas & $0.0006 \%$ & $\begin{array}{l}\text { valor_pens_invalid_men_ } \\
\text { mas_16_smlv }\end{array}$ & $0.0010 \%$ \\
\hline pobl_inactiva & $-0.0427 \%$ & valor_pens_vejez_men_2_smlv & $-0.0920 \%$ \\
\hline no_ocupado & $0.0752 \%$ & $\begin{array}{l}\text { valor_pens_vejez_men_2- } \\
\text { 4_smlv }\end{array}$ & $0.1047 \%$ \\
\hline ocupado & $-0.0325 \%$ & $\begin{array}{l}\text { valor_pens_vejez_men_4- } \\
7 \text { _smlv }\end{array}$ & $-0.0370 \%$ \\
\hline formal & $0.9183 \%$ & $\begin{array}{l}\text { valor_pens_vejez_men_7- } \\
\text { 10_smlv }\end{array}$ & $0.0193 \%$ \\
\hline \multirow[t]{3}{*}{ informal } & $-0.9183 \%$ & $\begin{array}{l}\text { valor_pens_vejez_men_10- } \\
13 \text { smlv }\end{array}$ & $0.0007 \%$ \\
\hline & & $\begin{array}{l}\text { valor_pens_vejez_men_13- } \\
\text { 16_smlv }\end{array}$ & $0.0017 \%$ \\
\hline & & $\begin{array}{l}\text { valor_pens_vejez_men_ } \\
\text { mas_16_smlv }\end{array}$ & $0.0025 \%$ \\
\hline
\end{tabular}

Fuente: elaboración propia. 
Modelación basada en agentes en el sistema pensional colombiano.

\section{Anexo 2.}

\section{Parámetros de variación:}

\begin{tabular}{|c|c|c|}
\hline Participacion $(\mathbf{P})$ & Nombre de la Variable & Participacion $(\mathbf{P})$ \\
\hline $5.92 \%$ & escolar_0-1 & $7.49 \%$ \\
\hline $49.47 \%$ & escolar_1-5 & $39.73 \%$ \\
\hline $50.53 \%$ & escolar_6-10 & $28.77 \%$ \\
\hline $44.90 \%$ & escolar_11 & $10.93 \%$ \\
\hline $23.00 \%$ & escolar_12+ & $13.08 \%$ \\
\hline $23.00 \%$ & rango_sal_men_2_smlv & $89.32 \%$ \\
\hline $4.20 \%$ & rango_sal_men_2-4_smlv & $6.09 \%$ \\
\hline $5.00 \%$ & rango_sal_men_4-7_smlv & $2.35 \%$ \\
\hline $2.27 \%$ & rango_sal_men_7-10_smlv & $0.84 \%$ \\
\hline $97.73 \%$ & rango_sal_men_10-13_smlv & $0.35 \%$ \\
\hline $95.84 \%$ & rango_sal_men_13-16_smlv & $0.26 \%$ \\
\hline $4.16 \%$ & rango_sal_men_mas_16_smlv & $0.79 \%$ \\
\hline $74.06 \%$ & cotiza & $42.20 \%$ \\
\hline $4.28 \%$ & no_cotiza & $57.80 \%$ \\
\hline $21.67 \%$ & cotiza_rpm & $51.66 \%$ \\
\hline $5.42 \%$ & cotiza_rais & $48.34 \%$ \\
\hline $94.58 \%$ & cambio_regimen & $6.78 \%$ \\
\hline $8.96 \%$ & no_cambio_regimen & $93.22 \%$ \\
\hline $8.84 \%$ & pensine & $2.27 \%$ \\
\hline $8.88 \%$ & no_pensione & $97.73 \%$ \\
\hline $9.02 \%$ & valor_pens_sobrv_men_2_smlv & $88.78 \%$ \\
\hline $8.91 \%$ & valor_pens_sobrv_men_2-4_smlv & $7.94 \%$ \\
\hline $8.21 \%$ & valor_pens_sobrv_men_4-7_smlv & $2.37 \%$ \\
\hline $7.35 \%$ & valor_pens_sobrv_men_7-10_smlv & $0.51 \%$ \\
\hline $6.65 \%$ & valor_pens_sobrv_men_10-13_smlv & $0.20 \%$ \\
\hline
\end{tabular}




\begin{tabular}{|c|c|c|}
\hline Participacion $(\mathbf{P})$ & Nombre de la Variable & Participacion $(\mathbf{P})$ \\
\hline $5.96 \%$ & valor_pens_sobrv_men_13-16_smlv & $0.09 \%$ \\
\hline $5.99 \%$ & valor_pens_sobrv_men_mas_16_smlv & $0.12 \%$ \\
\hline $5.57 \%$ & valor_pens_invalid_men_2_smlv & $96.06 \%$ \\
\hline $4.60 \%$ & valor_pens_invalid_men_2-4_smlv & $3.17 \%$ \\
\hline $3.58 \%$ & valor_pens_invalid_men_4-7_smlv & $0.61 \%$ \\
\hline $2.71 \%$ & valor_pens_invalid_men_7-10_smlv & $0.11 \%$ \\
\hline $1.92 \%$ & valor_pens_invalid_men_10-13_smlv & $0.02 \%$ \\
\hline $1.42 \%$ & valor_pens_invalid_men_13-16_smlv & $0.01 \%$ \\
\hline $1.43 \%$ & valor_pens_invalid_men_mas_16_smlv & $0.01 \%$ \\
\hline $33.12 \%$ & valor_pens_vejez_men_2_smlv & $70.31 \%$ \\
\hline $13.40 \%$ & valor_pens_vejez_men_2-4_smlv & $18.47 \%$ \\
\hline $53.48 \%$ & valor_pens_vejez_men_4-7_smlv & $7.32 \%$ \\
\hline $42.20 \%$ & valor_pens_vejez_men_7-10_smlv & $2.17 \%$ \\
\hline \multirow[t]{3}{*}{$57.80 \%$} & valor_pens_vejez_men_10-13_smlv & $0.95 \%$ \\
\hline & valor_pens_vejez_men_13-16_smlv & $0.50 \%$ \\
\hline & valor_pens_vejez_men_mas_16_smlv & $0.30 \%$ \\
\hline
\end{tabular}

Fuente: elaboración propia. 


\section{Referencias}

Arango Escobar, A., Echeverry Garzón, J. A., Merchán Hernández, C. \& Piraquive Galeano, G. (2001). Elementos para el debate sobre una nueva reforma pensional en Colombia. Bogotá: Departamento Nacional de Planeación - DNP.

Arias, M. \& Mendoza, J. C. (2009). Un modelo de simulación del régimen pensional de ahorro individual con solidaridad en Colombia. Bogotá: Banco de la Republica.

Ayala, U. \& Acosta, O. L. (2002). Políticas para promover una ampliación de la cobertura del sistema de pensiones en Colombia. Santiago de Chile: Cepal. Recuperado de http://repositorio.cepal.org/bitstream/handle/11362/5096/S2002613_es.pdf?sequence=1

Barr, N. (2006). Pensions: Overview of the issues. Oxford Review of Economic Policy, 22(1), $1-14$.

- (2001). La verdad sobre la reforma de los sistemas de pensiones. Finanzas y Desarrollo Fondo Monetario Internacional, 38(3), 6-9.

Betancur, J. (2000). Depreciación de las pensiones. Revista Facultad de Derecho y Ciencias Políticas, (102), 197-223.

Barr, N. \& Diamond, P. (2006). The economics of pensions. Oxford Review of Economic Policy, 22(1), 15-39.

Blake, D. (2006a). Pension finance. Londres: John Wiley \& Sons. . (2006b). Pension economics. Londres: John Wiley \& Sons.

Blake, D. \& Les M. (2006). On the sustainability of the UK State Pension System in the light of population ageing and declining fertility. The Economic fournal, 116(512), 286-305.

Brenes-Camacho, G. (2011). Favourable changes in economic well-being and self-rated health among the elderly. Social Science \& Medicine, 72(8), 1228-1235.

Bour, J. L. (1994). Reformas de los regímenes previsionales en América Latina. San José de Costa Rica: Editorial Comisión Nacional de Valores.

Carrillo Guarín, J. C. (2001). Evaluación y prospectiva del sistema general de pensiones en Colombia: análisis de su validez frente a la calidad de vida. Actualidad Laboral, (104), 5-17. 
ISSN: 0124-3551 / Año 16, No 24 / enero-junio / pp. 119-162

Clamorro Gómez, J. M. (2006). Privatización de las pensiones, garantía mínima y generaciones futuras: el caso español. Boletín de Estudios Económicos, 61(187), 91-110.

Clavijo, S. (2011). Sostenibilidad de mediano plazo del sistema de salud y pensiones. Debates de Coyuntura Económica. Bogotá: Fedesarrollo.

Conte, R., Hegselmann, R. \& Terna, P. (eds.). (1997). Simulating social phenomena. Lecture Notes in Economics and Mathematical Systems 456. Berlín: Springer-Verlag.

Edmonds, B., Moss, S. \& Davidsson, P. (2001). The use of models-making MABS actually work. En: Multi-agent-based simulation. Lecture Notes in Artificial Intelligence 1979 (pp. 15-32). Berlín: Springer-Verlag.

Emms, P. \& Haberman, S. (2008). Income drawdown schemes for a defined-contribution pension plan. The Journal of Risk and Insurance, 75(3), 739-761.

Esparza, E. J. (2004). El sistema previsional peruano y la necesidad de plantear una nueva reforma (Tesis de maestría, Pontificia Universidad Católica del Perú, Lima, Perú).

Franco, L. M. (2012). El sistema pensional colombiano: un análisis de los determinantes de la afiliación y su papel en el impacto de las reformas pensionales (Tesis de grado, Universidad del Valle, Cali, Colombia).

Galán, J. M., Izquierdo, L. R., Izquierdo, S. S., et al. (2009). Errors and artefacts in agent-based modelling. Fournal of Artificial Societies and Social Simulation, 12(1).

García-Florez, G. (2004). El debate actual sobre un sistema general de pensiones óptimo. Revista Equidady Desarrollo, (2), 107-120.

Gilbert, N. \& Troitzsch, K. G. (1999). Simulation for the social scientist. Buckingham: Open University Press.

Gilbert, N. \& Terna, P. (2000). How to build and use agent-based models in social science. Mind and Society, 1(1), 57-72.

Gotts, N., Polhill, J. \& Law, A.N.R. (2003). Agent-based simulation in the study of social dilemmas. Artificial Intelligence Review, 19(1), 3-92.

Herrera Córdoba, A. (1995). Regímenes de pensiones de capitalización individual. Bogotá: Comisión Nacional de Valores. Recuperado de http://www.cnbs.gob.hn/ files/seguros_pensiones/ssp_regpencapindiv.PDF

Hervas, J. R. \& Thomasz, E. O. (s. f.). ¿Constituye la economía evolutiva un nuevo paradigma epistemológico? Buenos Aires: Universidad de Buenos Aires. Recuperado 
de http://www.econ.uba.ar/www/institutos/epistemologia/marco_archivos/trabajos_XV_archivos/Garnica $\% 20 y \% 20$ Thomasz- $\% 20 \%$ BFConstituye $\% 201 \mathrm{l} \% 20$ econom $\% \mathrm{EDa} \% 20$ evolutiva $\% 20$ un $\% 20$ nuev.pdf

López Montaño, G. (2005). De trabajadores pobres a ancianos indigentes. Recuperado de http://www.cecilialopezcree.com/k_OldTopics/c_Nacional/2005/1_Trimestre /b_Ancianos.htm

Marco, F. (2004). Los sistemas de pensiones en América Latina: un análisis de género. Cuadernos de la Cepal, (90), 1-262. Recuperado de http://repositorio.cepal.org/ bitstream/handle/11362/27830/S0408643_es.pdf?sequence=1

Marie, O. \& Vall Castello, J. (2012). Measuring the (income) effect of disability insurance generosity on labour market participation. Fournal of Public Economic, 96(2), 198-210.

Mesa-Lago, C. (2004). Evaluación de un cuarto de siglo de reformas estructurales de pensiones en América Latina. Revista de la Cepal, (84), 54-82.

Moreno, Á. \& Ortiz, F. (2010). Economía política de la reforma del sistema colombiano de pensiones. Revista de Economía Institucional, 12(22), 167-192.

Moreno Cadavid, J. (2007). Modelo de simulación basado en agentes de software inteligentes para el aprendizaje de estrategias de comercialización de energía eléctrica en Colombia (Tesis de maestría, Universidad Nacional de Colombia, Bogotá, Colombia).

Novoa, M. \& Vargas, O. (2011). Aproximación a la informalidad en el mercado laboral: una modelación basada en agentes. Bogotá: Universidad Nacional de Colombia.

Núñez, F. (1999). El sistema pensional colombiano: régimen general, regímenes especiales y de transición. Bogotá: Legis Editores.

Ordax, J. M. (2008). Simulación basada en agentes en teoría de juegos evolutiva: ¿pueden las metanormas evitar el colapso de una norma social? Ponencia presentada en el IX Congreso de Ingeniería de Organización, Asociación para el Desarrollo de la Ingeniería (ADINGOR), Burgos, España.

Parra, J. C. (2001). Dnpension: un modelo de simulación para estimar el costo fiscal del sistema pensional colombiano. Archivos de Economía, Planeación y Desarrollo, 32(2), 255-294.

Pastrana, M., et al. (2005). Simulación basada en agentes inteligentes del proceso de negociación del mercado de energía eléctrica en Colombia. Bogotá: Universidad Nacional de Colombia. 
ISSN: 0124-3551 / Año 16, No 24 / enero-junio / pp. 119-162

Squazzoni, F. (2008). The micro-macro link in social simulation. Sociologica. Italian Journal of Sociology Online, (1).

Sánchez Martín, A. R. (2010). Endogenous retirement and public pension system reform in Spain. Economic Modelling, 27(1), 336-349.

Sánchez, R. M. (2008). La crisis del sistema pensional colombiano. Análisis de cobertura y críticas a las fallidas reformas. Ensayos de Economía, (32), 135-164.

Sánchez, R. (2013). Do reductions of standard hours affect employment transitions? Evidence from Chile. Labour Economics, (20), 24-37.

Santa María, M. (2010). El sistema pensional en colombia: retos y alternativas para aumentar la cobertura. Debates de Coyuntura Económica. Bogotá: Fedesarrollo.

Torsun, I.S. (1995) "Multiangent Systems", in Foundation of Intelligent Knoledge Based Systems. Academic Press, San Diego, C.A.

Uthoff, A. (2006). Brecha del Estado de Bienestar y reformas a los sistemas de pensiones en América Latina y el Caribe. Revista de la Cepal, (89), 1-55. Recuperado de http:// www.cepal.org/revista/noticias/articuloCEPAL/3/26353/LGG2312eUthoff.pdf

Williamson, J. B. \& Hochman, G. (1995). Innovative old-age security models for developing nations: Chile and Brazil. Fournal of Aging Studies, 9(3), 245-262.

Whitehouse, E. (2006). New indicators of 30 OECD countries' pension systems. Fournal of Pension Economics and Finance, 5(3), 275-298. 